\title{
Energy-efficient multi-pass turning operation using multi-objective backtracking search algorithm
}

\author{
Chao Lu, Liang Gao*, Xinyu Li, Peng Chen
}

State Key Lab of Digital Manufacturing Equipment \& Technology, School of Mechanical Science and Engineering, Huazhong University of Science and Technology, Wuhan, China

\begin{abstract}
Energy savings have become an essential consideration in sustainable manufacturing projects due to the associated environmental impacts and constraints on carbon emissions. In the past, machining operations primarily examined technological consideration (e.g., machining quality) and neglected energy consumption. Therefore, this paper investigates an energy-efficient multi-pass turning operation problem and establishes a multi-objective multi-pass turning operations model. Energy consumption and machining quality are both considered in this problem. Although several models of this problem have considered these criteria, the objectives are usually combined into a single objective using a weighted sum approach, which results in poor non-dominated solutions. To obtain high quality trade-offs between the two challenging objectives, a novel multi-objective backtracking search algorithm is proposed to solve this multi-objective optimization problem. To verify the feasibility and validity of the proposed algorithm, it is compared with other classical multi-objective metaheuristics on multi-objective multi-pass turning operations. This study's experimental results demonstrate that the proposed algorithm significantly outperforms other algorithms for this optimization problem, which is a significant result regarding practical application.
\end{abstract}

Keywords: Multi-objective evolutionary algorithm; Energy consumption; Parameter optimization; Backtracking search algorithm

\section{Introduction}

Energy savings have become a priority for the manufacturing industry in the past several decades. It is well-known that energy is one of the most important resources in the manufacturing sectors (Basar et al., 2013). Unfortunately, non-renewable sources, such as fossil oil, is gradually drying up whilst new energy sources have not been explored effectively. In this regard, more people have realized the importance of energy savings due to the rapid exhaustion of diverse non-renewable resources (Brundtland et al., 1987). For example, more than 170 countries reached an agreement on sustainable development and adopted environmental legislation in 1992 (Weiss, 1992). Moreover, energy consumption can generate carbon dioxide emissions, which leads to global warming. In China, $1 \mathrm{kWh}$ of electrical energy which mainly depends on coal power plant and hydro power plant can produce about $0.6747 \mathrm{~kg}$ of carbon dioxide (Li et al., 2013). Furthermore, manufacturing enterprises are responsible for about one-half of global energy consumption (Fang et al., 2011). As such, manufacturing sectors have become a major source of global warming, thus, their energy consumption is expected to be limited by higher taxes and regulatory legislation in the future. Given this challenge, it is necessary to search for practical methods to minimize energy consumption in manufacturing processes. Consequently, energy conservation and emissions reduction are attracting increasing attention. Recent related literatures on this topic of manufacturing production include (Guo et al., 2015; Lin et al., 2015; Liu et al., 2014; Wang et al., 2015).

One natural method for reducing energy consumption in the manufacturing process is to design power efficient

\footnotetext{
* Corresponding author.

E-mail address: gaoliang@ mail.hust.edu.cn (Liang Gao)
} 
machines (Mori et al., 2011) and embodied product energy frameworks (Kara et al., 2010; Rahimifard et al., 2010). The above approaches may require enormous financial investments, thus some companies may not select these approaches. Another interesting approach to reduce energy consumption is called the "machine turn on and off scheduling framework" (Mouzon et al., 2007). In this framework, energy consumption reductions are achieved by intelligently turning off machines instead of running them when idle. This approach has been used to address a single machine scheduling problem (Yildirim and Mouzon, 2012). Afterward, the framework was extended to the flexible flow shop scheduling problem for reducing energy consumption (Dai et al., 2013). However, this approach is not applicable for certain manufacturing sectors where the machines cannot be switched off during idle periods. Meanwhile, several researchers have observed that energy savings of up to $6 \%$ to $40 \%$ can be achieved with an optimal selection of such factors as cutting parameters, tools, and optimal tool path design. (Camposeco-Negrete, 2013; Velchev et al., 2014; Yan and Li, 2013). Furthermore, energy consumption during machining is responsible for a substantial part of the environmental burden (Rajemi et al., 2010). Therefore, it is highly important to reduce the energy consumption associated with machining parameter optimization.

This paper primarily investigates parameter optimization of multi-pass turning operations. This optimization problem has been one of the most widely studied optimization models in past decades (Chen and Tsai, 1996; Yildiz, 2012, 2013). However, little attention has been paid to energy consumption in this model. To fill this knowledge gap, the multi-pass turning optimization problem should simultaneously consider machining quality and energy consumption. Although energy consumption in AISI 6061 T6 turning operations has been researched (Camposeco-Negrete, 2013), the nature of this multi-objective optimization problem was neglected. That is, this issue is a typical multi-objective optimization problem (MOP). A traditional weighted sum method is usually used to optimize this problem, but this practice usually generates weak non-dominated solutions by performing multiple runs. To the best of the authors' knowledge, multi-objective evolutionary algorithms (MOEAs) can obtain non-dominated solutions in a single run and are suitable for MOPs. MOEAs have been successfully applied to parameter optimization problems (Ganesan et al., 2013; Gao et al., 2012; Solimanpur and Ranjdoostfard, 2009; $\mathrm{Wu}$ et al., 2012). Therefore, MOEAs are much more practical for improving the energy efficiency in the production systems and have attracted more and more research attention (Liu et al., 2014; Winter et al., 2014).

As we know, one of the state-of-the-art multi-objective metaheuristics is the non-dominated sorting genetic algorithm II (NSAG-II), which is the most effective multi-objective evolutionary algorithm (MOEA). However, this algorithm can be easily trapped into local optima when solving more complex MOPs, which leads to poor convergence and low efficiency. Therefore, designing an effective algorithm plays a key role in solving optimization problems. Recently, the backtracking search algorithm (BSA), which was invented by Civicioglu (2013), has been demonstrated to be a promising method for solving single objective optimization problems due to its high convergence speed and ease of implementation (Askarzadeh and Coelho, 2014; El-Fergany, 2015; Modiri-Delshad and Abd Rahim, 2014). Moreover, BSA outperforms other classical metaheuristics such as differential evolutionary (DE) and its derivatives, particle swarm optimizer (PSO), and artificial bee colony $(\mathrm{ABC})$. The main reason for the outperformance is that a dual population is utilized in BSA to maintain a good balance between exploration and exploitation. More precisely, one population is used to exploit better solutions, and the other, called the historical population, is adopted to explore new areas of the search space. At the same time, the main goal of this paper is to obtain optimal trade-offs between the energy consumption and machining quality closer to the true Pareto front. Based on the effectiveness of the BSA and nature of the MOP, a novel multi-objective backtracking search algorithm (MOBSA) is proposed to address the problem of multi-pass turning operations with the two conflicting objectives. To examine the performance of the proposed MOBSA, MOBSA is compared with other multi-objective evolutionary algorithms such as the NSGA-II (Deb et al., 2002), MOPSO (Sierra and Coello Coello, 2005), MOEA/D with constraints (Zapotecas Martinez and Coello Coello, 2014), and 
multi-objective harmony search (MOHS) (Li et al., 2015). The experimental results demonstrate the effectiveness of the MOBSA on multi-pass turning operations.

The paper is organized as follows. A formal definition of the problem is given in Section 2. In Section 3, a multi-objective backtracking search algorithm for the multi-pass turning optimization problem is proposed. The experimental results are presented and analyzed in Section 4. Conclusions and future work are presented in Section 5 .

\section{Mathematical model of multi-pass turning operations}

The characteristic of the multi-pass turning model is nonlinear and highly constrained, which restricts the application of traditional gradient based methods. Therefore, metaheuristics are very suitable for solving this complex problem. In multi-pass turning operations, the main objective of the problem is to obtain trade-offs between unit production energy consumption and machining quality (or precision). The optimization problem contains crucial optimization variables, such as the cutting speed, feed rate, depth of cut and number of rough cuts, which affect both the energy consumption and precision value.

\subsection{Nomenclature}

The notations used throughout the paper are as follows:

\begin{tabular}{cll}
\hline Parameters & & \\
$A_{1} \quad A_{2}$ & coefficient pertaining to idle load power & \\
$C_{0}$ & constant pertaining to tool-life equation & \\
$C_{F c}$ & main cutting force coefficient & \\
$d_{r L}$ & lower bound of depth of rough cut & $(\mathrm{mm})$ \\
$d_{r U}$ & upper bound of depth of rough cut & $(\mathrm{mm})$ \\
$d_{S L}$ & lower bound of depth of finish cut & $(\mathrm{mm})$ \\
$d_{S U}$ & upper bound of depth of finish cut & $(\mathrm{mm})$ \\
$d_{t}$ & total depth of metal to be removed & $(\mathrm{mm})$ \\
$D$ & diameter of workpiece & $(\mathrm{mm})$ \\
$D_{i}$ & waiting process surface diameter in the $i$-th pass & $(\mathrm{mm})$ \\
$E$ & elastic modulus & $(\mathrm{MPa})$ \\
$f_{r L}$ & lower bound of feed in rough machining & $(\mathrm{mm} / \mathrm{rev})$ \\
$f_{r U}$ & upper bound of feed in rough machining & $(\mathrm{mm} / \mathrm{rev})$ \\
$f_{S L}$ & lower bound of feed in finish machining & $(\mathrm{mm} / \mathrm{rev})$ \\
$f_{S U}$ & upper bound of feed in finish machining & $(\mathrm{mm} / \mathrm{rev})$ \\
$F_{r}$ & cutting force during rough machining & $(\mathrm{kgf})$ \\
$F_{S}$ & cutting force during finish machining & $(\mathrm{kgf})$ \\
$F_{U}$ & maximum allowable cutting force & $(\mathrm{kgf})$ \\
$h_{1}$ & constant pertaining to tool travel and approach & $(\mathrm{min} / \mathrm{mm})$ \\
$h_{2}$ & constant pertaining to tool travel and depart time & $(\mathrm{min})$ \\
$J$ & moment of inertia & \\
$k_{2} \quad k_{3}$ & constants for roughing and finishing parameter relations \\
$k_{f}$ & coefficient pertaining to specific tool-workpiece combination & \\
$k_{q}$ & coefficient pertaining to equation of chip-tool interface temperature & \\
$K$ & clamping method coefficient & \\
$K_{F c}$ & correction coefficient & \\
$L$ & length of workpiece & \\
& & \\
&
\end{tabular}




\begin{tabular}{|c|c|c|}
\hline$n_{i}$ & rotational speed after the $i$-th work step when machine is idle & $(\mathrm{r} / \mathrm{min})$ \\
\hline$n_{L}$ & lower number of rough passes & \\
\hline$n_{U}$ & upper number of rough passes & \\
\hline$p$ & constant pertaining to the tool-life equation & \\
\hline$n_{t}$ & the edge piece & \\
\hline$P_{r}$ & cutting power during roughing & $(\mathrm{kW})$ \\
\hline$P_{S}$ & cutting power during finishing & $(\mathrm{kW})$ \\
\hline$P_{U}$ & maximum allowable cutting power & $(\mathrm{kW})$ \\
\hline$P_{0}$ & power when replacement tool or loading and unloading operations & $(\mathrm{kW})$ \\
\hline$P_{u}$ & the idle power of machine due to tool idle motion & $(\mathrm{kW})$ \\
\hline$P_{w}$ & tool energy per cutting edge & (MJ/insert) \\
\hline$q$ & constant pertaining to the tool-life equation & \\
\hline$Q_{r, i}$ & temperature during the $i$-th pass roughing & $\left({ }^{\circ} \mathrm{C}\right)$ \\
\hline$Q_{s}$ & temperature during finishing & $\left({ }^{\circ} \mathrm{C}\right)$ \\
\hline$Q_{U}$ & maximum allowable temperature & $\left({ }^{\circ} \mathrm{C}\right)$ \\
\hline$r$ & constant pertaining to the tool-life equation & \\
\hline$R_{a}$ & maximum allowable surface roughness & $(\mathrm{mm})$ \\
\hline$R_{n}$ & nose radius of cutting tool & $(\mathrm{mm})$ \\
\hline$S_{c}$ & limit of stable cutting region & \\
\hline$t_{c}$ & constant term of loading and unloading operations & $(\min )$ \\
\hline$t_{e}$ & tool exchange time & $(\mathrm{min} / \mathrm{edge})$ \\
\hline$t_{i}$ & total idle time & $(\min )$ \\
\hline$t_{m}$ & total machining time & $(\min )$ \\
\hline$t_{m r}$ & machining time for $n$ roughing passes & $(\min )$ \\
\hline$t_{m s}$ & machining time for one finishing pass & $(\min )$ \\
\hline$t_{r}$ & a variable time due to tool motion & $(\min )$ \\
\hline$T_{c}$ & replacement cycle of cutting fluid & (month) \\
\hline$T_{p}$ & tool life considering roughing and finishing & $(\min )$ \\
\hline$T_{r}$ & tool life for roughing & $(\min )$ \\
\hline$T_{S}$ & tool life for finishing & $(\min )$ \\
\hline$T_{L}$ & lower bound of tool life & $(\min )$ \\
\hline$T_{U}$ & upper bound of tool life & $(\min )$ \\
\hline$V_{r L}$ & lower bound of cutting speeds in rough machining & $(\mathrm{m} / \mathrm{min})$ \\
\hline$V_{r U}$ & upper bound of cutting speeds in rough machining & $(\mathrm{m} / \mathrm{min})$ \\
\hline$V_{S L}$ & lower bound of cutting speeds in finish machining & $(\mathrm{m} / \mathrm{min})$ \\
\hline$V_{S U}$ & upper bound of cutting speeds in finish machining & $(\mathrm{m} / \mathrm{min})$ \\
\hline$w_{r}$ & rotational speed during rough machining & $(\mathrm{r} / \mathrm{min})$ \\
\hline$w_{s}$ & rotational speed during finish machining & $(\mathrm{r} / \mathrm{min})$ \\
\hline$x_{F c}$ & back cutting depth exponent & \\
\hline$x_{e}$ & embodied energy of auxiliary material & $(\mathrm{KJ} / \mathrm{kg})$ \\
\hline$y_{F C}$ & feed rate exponent & \\
\hline$\theta$ & weighted coefficient of tool life for roughing and finishing & \\
\hline$\rho_{a}$ & density of auxiliary material & $(\mathrm{kg} / \mathrm{L})$ \\
\hline$\delta$ & constant pertaining to expression of chip-tool interface temperature & \\
\hline
\end{tabular}




\begin{tabular}{cll}
\hline$\tau$ & constant pertaining to expression of chip-tool interface temperature & \\
$\varphi$ & constant pertaining to expression of chip-tool interface temperature & \\
$\mu$ & constant of cutting force equation & \\
$\vartheta$ & constant of cutting force equation & \\
$\lambda$ & constant pertaining to expression of stable cutting region & \\
$v$ & constant pertaining to expression of stable cutting region & \\
$v_{a}$ & consumption velocity of auxiliary material & $(\mathrm{L} / \mathrm{min})$ \\
$\eta$ & power efficiency & \\
$\eta_{F c}$ & cutting speed exponent & \\
$\eta_{j}$ & ratio between back force and main cutting force & $(\mathrm{L})$ \\
$\Delta \mathrm{v}$ & additional cutting fluid volume & $(\mathrm{L})$ \\
$\mathrm{v}_{0}$ & initial cutting fluid volume & \\
$\delta_{a}$ & concentration of cutting fluid & $(\mathrm{mm})$ \\
Variables & & $(\mathrm{mm})$ \\
$d_{r, i}$ & the depth of cut for the $i$-th rough pass & $(\mathrm{mm} / \mathrm{rev})$ \\
$d_{S}$ & the depth of cut for finish machining & $(\mathrm{mm} / \mathrm{rev})$ \\
$f_{r, i}$ & feed for the $i$-th pass of rough machining & $(\mathrm{m} / \mathrm{min})$ \\
$f_{S}$ & feed in finish machining & $(\mathrm{m} / \mathrm{min})$ \\
$n$ & number of rough passes &
\end{tabular}

\subsection{Objective functions}

For this study, the problem is considered to have two objectives: unit production energy consumption and machining precision.

\subsubsection{Minimization of energy consumption}

The first objective is called unit production energy consumption. Energy consumption is difficult to compute or predict, because it includes machining energy, idle energy, tool energy, etc. However, it mainly contains five forms of energy consumption as follows:

- Energy consumption of machining $\left(E C_{1}\right)$ : energy required for multi-pass roughing and finishing.

- Energy consumption during machine idle $\left(E C_{2}\right)$ : machine idle energy consumed for preparation work (i.e., loading and unloading operation) and tool motion.

- Energy consumption for tool replacement $\left(E C_{3}\right)$ : energy consumed when tools are replaced.

- Energy consumption due to tool wear $\left(E C_{4}\right)$ : energy consumption required for tool wear.

- Energy consumption of the cutting fluid $\left(E C_{5}\right)$ : energy consumption required to prepare cutting fluid and deal with waste liquid.

For the sake of convenient calculations, the total energy consumption is computed from the five forms of energy consumption listed above in this paper. Therefore, the first objective of this problem can be defined as follows:

$$
\min f_{1}=E C_{1}+E C_{2}+E C_{3}+E C_{4}+E C_{5}
$$

(1) Machining energy consumption $\left(E C_{1}\right)$ 
In this work, the turning process is considered to occur at the constant rotational speeds for rough and finish machining. However, the constant rotational speeds for rough and finish operations are different. Turning operations consumed energy for multi-pass roughing and finishing operations. The total machining time $t_{m}$ is composed of $n$ roughing passes $\left(t_{m r}\right)$ and one finishing pass $\left(t_{m s}\right) . t_{m}$ is written as follows (Chauhan et al., 2015; Shin and Joo, 1992).

$$
t_{m}=t_{m r}+t_{m s}
$$

The machining process time during roughing $t_{m r}$ is defined as:

$$
t_{m r}=\sum_{i=1}^{n} \frac{\pi D_{i} L}{1000 V_{r, i} f_{r, i}}=\sum_{i=1}^{n} \frac{L}{w_{r} f_{r, i}}
$$

where $V_{r, i}$ and $f_{r, i}$ denote the cutting speed and feed rate in the $i$-th roughing pass, respectively. Note that $V_{r, i}$ and $f_{r, i}$ of each roughing pass are different. $D_{i}$ represents the waiting process surface diameter of the workpiece in the $i$-th roughing pass. Namely, $D_{1}=D ; D_{i}=D_{i-1}-2 d_{r, i-1}, i=\{2, \ldots, n\}$, where $d_{r, i}$ is the depth of cut for the $i$-th pass of rough machining.

The machining process time during finishing $t_{m s}$ is formulated as:

$$
t_{m s}=\frac{\pi D_{n+1} L}{1000 V_{s} f_{s}}=\frac{L}{w_{s} f_{s}}
$$

where $D_{n+1}$ represents the waiting process surface diameter in the final pass (i.e., finishing pass). Namely, $D_{n+1}=D_{n}-2 d_{r, n}$.

Thus, the energy consumption for roughing and finishing is:

$$
E C_{1}=P_{r, i} \sum_{i=1}^{n} \frac{L}{60 * 1000 V_{r, i} f_{r, i}}+P_{s} \frac{\pi D_{n+1} L}{60 * 1000 V_{s} f_{s}}=P_{r, i} \sum_{i=1}^{n} \frac{L}{60 * w_{r} f_{r, i}}+P_{s} \frac{L}{60 * w_{s} f_{s}}
$$

where $P_{r, i}=\frac{k_{f} f_{r, i}^{\mu} d_{r, i}^{\vartheta} V_{r, i}}{6120 \eta}$ and $P_{S}=\frac{k_{f} f_{s}^{\mu} d_{s}^{\vartheta} V_{s}}{6120 \eta}$ (Chauhan et al., 2015).

(2) Energy consumption for machine idle $\left(E C_{2}\right)$

The machine idle time $\left(t_{i}\right)$ can be divided into a constant time $\left(t_{c}\right)$ due to loading and unloading operations and a variable time $\left(t_{r}\right)$ due to tool idle motion (Shin and Joo, 1992). The total idle time can be expressed as

$$
t_{i}=t_{c}+t_{r}
$$

The variable time $\left(t_{r}\right)$ is written as follows (An, 2003):

$$
t_{r}=\left(h_{1} L+h_{2}\right)(n+1)
$$


Therefore, the energy consumption for idle time is:

$$
E C_{2}=\frac{1}{60}\left(P_{u}\left(h_{1} L+h_{2}\right)(n+1)+P_{0} t_{c}\right)
$$

In actual machining, the idle power of the machine tool is closely related to the length of the transmission line, the condition of lubrication and the spindle speed. Assuming that the transmission line and lubrication do not change, the idle power of the machine tool is a quadratic function of the spindle rotational speed, the equation is expressed as $P_{u}=10^{-3} \times\left(40.6+A_{1} n_{i}+A_{2} n_{i}^{2}\right)$, where $n_{i}$ is the spindle rotational speed when machine is idle, but is a constant $\left(n_{i}=\frac{10^{3} V_{i}}{\pi D_{i}}, V_{i}\right.$ represents finishing or roughing cut speed) in this paper. Detailed information for this equation can be found in (Li et al., 2013; Shi et al., 2009).

(3) Energy consumption for tool replacement $\left(E C_{3}\right)$

The energy consumption for tool replacement can be expressed as (Ri-yao, 1984):

$$
E C_{3}=\frac{P_{0} t_{e}\left(\frac{t_{m}}{T_{p}}\right)}{60}
$$

The wear rate of tools differs for the roughing and finishing operations due to the various machining conditions. The tool life in such a case can be expressed as (Chen and Tsai, 1996; Onwubolu and Kumalo, 2001):

$$
T_{p}=\theta T_{r}+(1-\theta) T_{s}
$$

where

$$
\begin{gathered}
T_{r}=\sum_{i=1}^{n} \frac{c_{O}}{V_{r, i}^{p} f_{r, i}^{q} d_{r, i}^{r}} \\
T_{S}=\frac{c_{O}}{V_{s}^{p} f_{s}^{q} d_{s}^{r}}
\end{gathered}
$$

(4) Energy consumption for tool wear $\left(E C_{4}\right)$

Energy consumption for tool wear can be defined as the energy footprint of the tool per edge piece. This consumption denotes the energy embodied in the tool and is given as (Arif et al., 2013):

$$
E C_{4}=P_{w}\left(\frac{t_{m}}{T_{p}}\right) n_{t}
$$

where $\frac{t_{m}}{T_{p}}$ is the number of cutting edges per piece, $n_{t}$ is the edge piece in the above formula, $n_{t}$ is set to 1 in this paper.

(5) Energy consumption for cutting fluid $\left(E C_{5}\right)$

The cutting fluid energy can be computed based on the specific embodied energy of the cutting fluid and the volume it occupies. The energy consumption for water-based cutting fluid mainly includes two parts: (1) energy consumption for preparing pure mineral oil such as the paraffin; (2) energy consumption for dealing with waste 
liquid. The energy consumption by the cutting fluid is defined as follows (Li et al., 2013; Wang et al., 2014; Yun, 2014):

$$
E C_{5}=\frac{t_{m+} t_{i+} t_{e}}{43200 T_{c}} x_{e} \rho_{a}\left[\left(\mathrm{v}_{0}+\Delta \mathrm{v}\right)+\left(\mathrm{v}_{0}+\Delta \mathrm{v}\right) / \delta_{a}\right]
$$

Note that $T_{c}$ represents the life-cycle of the cutting fluid and its unit is month, which requires to be translated into the uniform unit "min" using multiplying by 43200 .

According to Eq. (1), the first objective for optimization can be written as:

$$
\begin{array}{r}
\min f_{1}=\left[\frac{1}{60} P_{r} t_{m r},+\frac{1}{60} P_{s} t_{m s}+\frac{1}{60}\left(P_{u}\left(h_{1} L+h_{2}\right)(n+1)+P_{0} t_{c}\right)+P_{0} t_{e}\left(\frac{t_{m}}{60 T_{p}}\right)+P_{w}\left(\frac{t_{m}}{T_{p}}\right)+\frac{t_{m+} t_{i+} t_{e}}{43200 T_{c}} x_{e} \rho_{a}\left[\left(\mathrm{v}_{0}+\right.\right.\right. \\
\left.\left.\Delta \mathrm{v})+\left(\mathrm{v}_{0}+\Delta \mathrm{v}\right) / \delta_{a}\right]\right] \quad(15)
\end{array}
$$

\subsubsection{Minimization of machining precision value}

The second objective is to minimize the machining precision (accuracy) of the workpiece. The smaller this objective value is, the better the quality of machining production is. Details about this criterion can be found in (Wang, 2006; Yang, 2011). The second objective can be written as follows:

$$
\min f_{2}=\frac{10^{3} C_{F c} c_{s}^{x F c} f_{s}^{y F c} V_{s}^{\eta F c} K_{F c} L^{3} \sqrt{1+\eta_{j}^{2}}}{K E J}
$$

where

$$
J=\frac{\pi\left(D-2 \sum_{i=1}^{n} d_{r, i}\right)^{4}}{64}
$$

The formulation has two challenging objectives. Thus, the final result of this problem is no unique optimal solution, but a set of solutions called "non-dominated solutions" or "compromise solutions", which are optimal trade-off solutions between both objectives.

\subsection{Parameter bounds and cutting condition constraints}

The optimal cutting parameters should satisfy a number of constraints. The above two objectives are imposed by various constraints: (1) the parameter limits on the depth of cut, cutting speed and feed rate as well as the (2) tool-life, (3) cutting force, (4) power, (5) stable cutting region, (6) chip-tool interface temperature, (7) surface finish, and (8) parameter relations constraints. These constraints are described in the following subsections.

2.3.1 Rough machining

$$
\begin{array}{ll}
\text { Depth of rough cut } & d_{r L} \leq d_{r, i} \leq d_{r U} \\
\text { Feed of roughing } & f_{r L} \leq f_{r, i} \leq f_{r U} \\
\text { Rough cutting speed } & V_{r L} \leq V_{r, i} \leq V_{r U} \\
\text { Tool-life constraint } & T_{L} \leq T_{r} \leq T_{U}
\end{array}
$$

In constraint (21), the tool life ( $\mathrm{min}$ ) for roughing is defined by Eq. (11). Considering the production energy consumption and quality of the machined part, the tool life should be within an acceptable range.

$$
\begin{array}{ll}
\text { Cutting force constraint } & F_{r, i}=k_{f} f_{r, i}^{\mu} d_{r, i}^{\vartheta} \leq F_{U} \\
\text { Power constraint } & P_{r, i}=\frac{k_{f} f_{r, i}^{\mu} d_{r, i}^{\vartheta} i_{r, i}}{6120 \eta} \leq P_{U}
\end{array}
$$


Stable cutting region constraint $\quad V_{r, i}^{\lambda} f_{r, i} d_{r, i}^{v} \geq S c$

Chip-tool interface temperature constraint $Q_{r, i}=k_{q} V_{r, i}^{\tau} f_{r, i}^{\varphi} d_{r, i}^{\delta} \leq Q_{U}$

2.3.2 Finish machining

$$
\begin{array}{ll}
\text { Depth of finish cut } & d_{S L} \leq d_{s} \leq d_{S U} \\
\text { Feed of finishing } & f_{S L} \leq f_{s} \leq f_{S U} \\
\text { Finish cutting speed } & V_{S L} \leq V_{S} \leq V_{S U} \\
\text { Tool-life constraint } & T_{L} \leq T_{S} \leq T_{U}
\end{array}
$$

In constraint (29), the tool life (min) for finishing is defined by Eq. (12). Considering the production energy consumption and quality of the workpiece, the tool life should be within an acceptable range. The other finishing constraints are as follows (Chen and Tsai, 1996; Yildiz, 2012, 2013).

$$
\begin{array}{ll}
\text { Cutting force constraint } & k_{f} f_{s}^{\mu} d_{s}^{\vartheta} \leq F_{U} \\
\text { Power constraint } & \frac{k_{f} f_{s}^{\mu} d_{s}^{\vartheta} V_{s}}{6120 \eta} \leq P_{U}
\end{array}
$$

Chip-tool interface temperature constraint $\quad k_{q} V_{s}^{\tau} f_{s}^{\varphi} d_{s}^{\delta} \leq Q_{U}$

Surface finish constraint

$$
\frac{f_{s}^{2}}{8 R_{n}} \leq R_{a}
$$

\subsubsection{Parameter relations}

In addition to all the constraints mentioned above, the relations between the parameters such as the speed, feed rate and depth of cut during the roughing and finishing processes should be given. They can be defined as (Chauhan et al., 2015; Chen, 2004; Shin and Joo, 1992):

$$
\begin{aligned}
& V_{s}>k_{1} V_{r, i} \\
& f_{r, i}>k_{2} f_{s} \\
& d_{r, i}>k_{3} d_{s} \\
& d_{t}=d_{s}+\sum_{i=1}^{n} d_{r, i}
\end{aligned}
$$

The total depth of cut $\left(d_{t}\right)$ is sum of the depth of the finish cut $\left(d_{s}\right)$ and total depth of the rough cut. The optimization method does not determine the optimal depth of the rough cut because it can be determined by the mathematical manipulation as expressed in Eq. (38). Therefore, the equality constraint (Eq. (38)) and design variable $\left(d_{s}\right)$ in the optimization procedure can be eliminated. In other words, the variable parameters $\left(V_{r, i}, f_{r, i}, d_{r, i}, V_{s}, f_{s}, n\right)$ are used to determine the trade-off approximations between the two objectives (i.e., energy consumption and machining precision).

\section{The proposed multi-objective metaheuristic}

In this section, the main step of the original BSA is outlined, and then a novel multi-objective algorithm MOBSA is presented for parameter optimization of multi-pass turning operations.

\subsection{Backtracking Search Algorithm}

The main steps of the BSA can be divided into five processes: initialization, selection-1, mutation, crossover and selection-II. A general BSA structure is presented in Fig. 1. A detailed description of the aforementioned steps is given in (Civicioglu, 2013); and the following subsections give a brief explanation. 


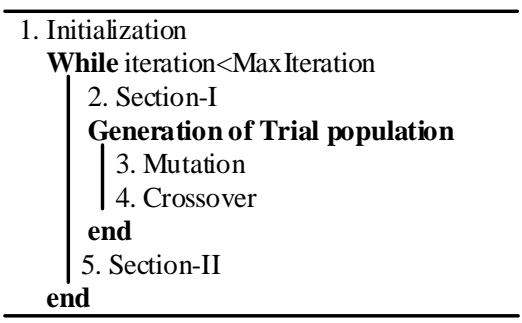

Fig. 1 A general structure of BSA (Civicioglu, 2013)

\subsubsection{Initialization}

BSA initializes the population $P$ as follows:

$$
P_{i, j}=\text { lower }_{j}+\left(\text { upper }_{j}-\text { lower }_{j}\right) \times \text { random } i=(1,2, \ldots, P S), j=(1,2, \ldots, P D)
$$

where $P S$ and $P D$ are the population size and problem dimension, respectively and random is a real value uniformly distributed between 0 and 1 . lower $_{j}$ and upper $_{j}$ represent the lower and upper bound in the $j$-th element of the $i$-th individual, respectively.

\subsubsection{Selection-I}

The BSA's Selection-I stage determines the historical population oldP to calculate the search direction. The initial historical population is determined by the following equation:

$$
\operatorname{oldP}_{i, j}=\text { lower }_{j}+\left(\text { upper }_{j}-\text { lower }_{j}\right) \times \text { random } i=(1,2, \ldots, P S), j=(1,2, \ldots, P D)
$$

The BSA has the option to generate oldP at the beginning of each iteration through the following equation:

$$
\text { if } a<b \text { then oldP }:=P \mid a, b \in[0,1]
$$

where $:=$ is the update operation. The above equation guarantees that the BSA can have a population randomly selected historical population and remembers this historical population until it is changed. After oldP is determined, a random permutation is used to randomly change the order of the individuals in oldP.

\subsubsection{Mutation}

The initial form of the trial population through mutation operation is as follows:

$$
T=P+(\text { old } P-P) \times F
$$

where $F$ is a scale factor which controls the amplitude of the search-direction matrix (oldP $-P$ ). In this paper, $F=3 \cdot$ random, where random is a real number uniformly distributed in $[0,1]$. Since the historical population is employed in the calculation of the search-direction matrix, the BSA takes advantages of its experiences from previous generations to obtain a trial population.

\subsubsection{Crossover}

The final trial population $T$ is generated by crossover in the BSA. The initial value of the trial population is called Mutant and is obtained in the mutation process. This search direction is guided by the trial individuals with better fitness values for the optimization problem. The crossover of the BSA works as follows. A binary integer-valued matrix (map) of size $N \times D$ is computed in the first step. This implies that the individuals of $T$ are generated by using the relevant individuals of $P$. If $\operatorname{map}_{i, j}=1, T$ is updated with $T_{i, j}:=P_{i, j}$. 


\subsubsection{Selection-II}

In the phase of the Selection-II, the $T_{i}$ that outperforms the corresponding $P_{i}$ in terms of fitness value is used to update the $P_{i}$. When the best solution $P_{\text {best }}$ dominates the previous global optimal value found by the BSA, the global optimal solution is replaced by $P_{\text {best }}$ and the global optimal value is also updated to be the fitness value of $P_{\text {best }}$. The principle of the BSA is rather simple. Therefore, BSA is easily applied to a variety of optimization problems (Civicioglu, 2013).

\subsection{The proposed MOBSA for multi-pass turning operations}

As previously mentioned, parameter optimization of multi-pass turning operations involves several conflicting objectives (i.e., energy consumption and machining precision) and is a typical multi-objective optimization problem (MOP). Without loss of generality, a MOP can be defined in the following form:

$$
\begin{gathered}
\min f(\mathbf{x})=\min \left[f_{1}(\mathbf{x}), f_{2}(\mathbf{x}), \ldots f_{m}(\mathbf{x})\right] \\
\mathbf{x}=\left(x_{1}, x_{2}, \ldots x_{n}\right) \in R^{n} \\
\text { s.t. }\left\{\begin{array}{l}
g_{i}(\mathbf{x}) \geq 0, i=1, \ldots, k \\
h_{j}(\mathbf{x})=0, j=1, \ldots, p
\end{array}\right.
\end{gathered}
$$

where $\mathbf{x}$ is called a decision vector and $R^{n}$ is the solution space. $f(\mathbf{x})$ constitutes $m$ individual objective functions.

For $\boldsymbol{a}$ and $\boldsymbol{b} \in R^{n}$, a vector $\boldsymbol{a}=\left[\mathrm{a}_{1}, \mathrm{a}_{2}, \ldots, \mathrm{a}_{\mathrm{n}}\right]^{\mathrm{T}}$ is said to dominate another vector $\boldsymbol{b}=\left[\mathrm{b}_{1}, \mathrm{~b}_{2}, \ldots, \mathrm{b}_{\mathrm{n}}\right]^{\mathrm{T}}($ denoted by $\boldsymbol{a} \prec \boldsymbol{b})$ if and only if $f_{i}(\boldsymbol{a}) \leq f_{i}(\boldsymbol{b})$ for each $i \in\{1, \cdots, m\}$ and $f_{j}(\boldsymbol{a})<f_{j}(\boldsymbol{b})$ for at least index $j \in\{1, \cdots, m\}$. A solution $\mathbf{x}^{*} \in R^{n}$ is called a Pareto optimal solution if there is not any a solution $\mathbf{x} \in R^{n}$ that dominates $\mathbf{x}^{*}$. The corresponding objective function is called Pareto optimal front vector $f\left(\mathbf{x}^{*}\right)$. For a Pareto optimal solution, the improvement of any objective must result in the deterioration of at least another objective. The set of all Pareto optimal solutions is called Pareto set $\left(S^{*}\right)$, while the set of all Pareto optimal front vectors is called the Pareto optimal front $\left(P F^{*}\right)$. The main goal of multi-objective optimization is to find $P F^{*}$. However, in general a Pareto front consists of a large number of points. Thus, a good Pareto front should contain a limited number of points which should be as close as possible to the $P F^{*}$ and should be uniformly spread as well. Therefore, a multi-objective backtracking search algorithm (MOBSA) is proposed to solve this parameter optimization of multi-pass turning operations.

The proposed MOBSA algorithm consists of 3 steps summarized as follows:

\section{Input.}

- A stopping condition;

- $\quad P S$ : population size;

- Input other parameters;

Output. The optimal results obtained;

Step 1) Initialization. Generate an initial population of $P S$ solutions according to the encoding scheme as in Section 3.1.1. The individual $i(i=1, \ldots, P S)$ in the population is a potential solution denoted by a vector $P_{i}=$ $\left[V_{r, 1}, \ldots, V_{r, n}, f_{r, 1} \ldots, f_{r, n}, d_{r, 1} \ldots, d_{r, n}, V_{s}, f_{s^{\prime}}, n\right] . P_{i, j}$ is the $j$-th element of the individual $i$, where $j=1, \ldots, 3 n+3$. The population is composed of all the vectors.

Step 2) Stopping condition. If the stopping criterion is satisfied, then stop and output the non-dominated solutions. Otherwise, go to step 3 as below.

\section{Step 3) Update.}

Step 3.1) Selection-I. Generate a historical population with size $P S$ and choose randomly a population from the dual population (i.e., initial population and historical population) as a parent population, as described in Section 


\subsection{2.}

Step 3.2) Mutation. This operator can create the initial form of the trial population. Section 3.1.3 gives details on mutation operation.

Step 3.3) Crossover. This step is to generate the final trial population at each iteration. More details can be found in Section 3.1.4. Note that the boundary constraints of real numbers are implemented through a simple approach: if $P_{i, j}^{t+1}>P_{i, j}^{+}$then $P_{i, j}^{t+1}=P_{i, j}^{+}$. If $P_{i, j}^{t+1}<P_{i, j}^{-}$then $P_{i, j}^{t+1}=P_{i, j}^{-} . P_{i, j}^{-}$and $P_{i, j}^{+}$represent the lower and upper bounds of the $j$-th element of individual $i$.

Step 3.4) Selection-II. This phase of the MOBSA is different from that of the basic BSA when solving MOPs, the newly generated trial solutions are evaluated in terms of both fitness values considered (i.e., energy consumption $f_{1}$ and machining precision $f_{2}$ ). In particular, all the solutions in the final population are sorted according to the non-dominated sorting technique, which favors simultaneously the population diversity and the convergence toward Pareto optimal solutions. Thus, each solution is associated with a rank equal to its non-dominance level as shown in Fig. 2 (e.g., 1 for the best level, 2 for the next best level, and so on). Then, within each front a specific crowding distance strategy is employed to define an ordering among individuals. The crowding distance is an indicator of the density of the solutions surrounding a current solution and can be obtained by calculating the sum of distances to the closest individual along each objective. That is, the crowding distance $c d_{i}$ of individual $i$ is $c d_{i}=\sum_{j=1}^{m}\left|f_{j}(i+1)-f_{j}(i-1)\right|$, where $m$ is the number of objectives, $f_{j}$ is the $j$-th objective function, the crowding distance of the extreme point is set to infinite. To achieve wide spread Pareto fronts (solutions differently balancing energy consumption and machining precision), individuals with a large crowding distance are superior to those with a small one for solutions that belong to the same non-dominated level. The newly generated population $Q_{t}$ is combined with existing parent population $P_{t}$ to form $Q_{t} \cup P_{t}$ solution vectors. Then non-dominated sorting strategy and crowding distance technique are performed on the combined population $Q_{t} \cup P_{t}$. Finally the best population with size $P S$ is selected from the combined population based on ranking and crowding distance for the next update as presented in Fig. $\mathbf{3}$.

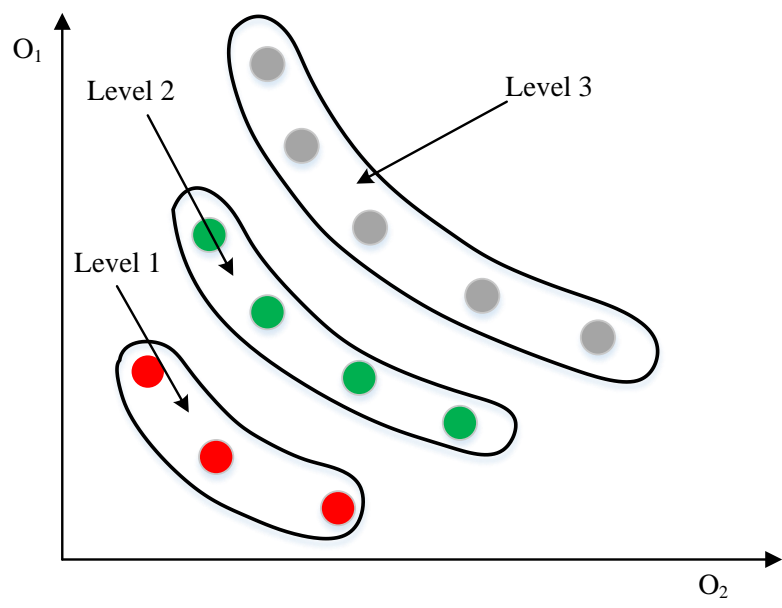

Fig. 2 Non-dominated levels 


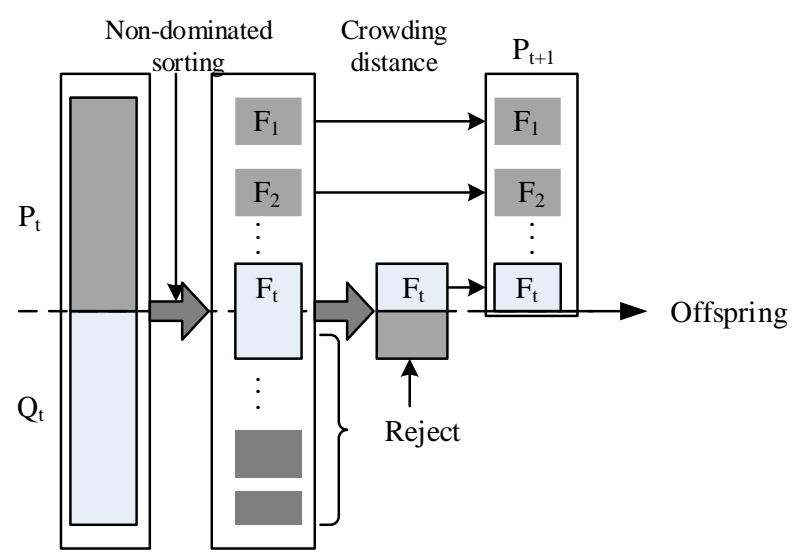

Fig. 3 Construction of population $\mathrm{P}_{\mathrm{t}+1}$

Note that a constraint handling mechanism is incorporated into MOBSA to deal with the constrained MOPs. This mechanism is the same as in (Deb et al., 2002; Tang and Wang, 2013). For solutions $\boldsymbol{a}$ and $\boldsymbol{b}, \boldsymbol{a}$ is said to constrained-dominate the solution $\boldsymbol{b}$, if any of the following conditions is met.

1. If the solution $\boldsymbol{a}$ is feasible and solution $\boldsymbol{b}$ is infeasible.

2. If solutions $\boldsymbol{a}$ and $\boldsymbol{b}$ are both feasible, and $\boldsymbol{a}$ dominates $\boldsymbol{b}$.

3. If solutions $\boldsymbol{a}$ and $\boldsymbol{b}$ are both infeasible, but solution $\boldsymbol{a}$ has a smaller overall constraint violation.

\section{Case study}

\subsection{Background of the optimization problem}

To assess the performance of the proposed MOBSA, the MOBSA is compared with other state-of-the-art MOEAs for solving the multi-pass turning operations. To implement the optimization problem, certain parameters should be provided in advance. The workpiece material of the optimization case belongs to a $\mathrm{C} 45$ carbon steel forging bar $80 \mathrm{~mm}(D)$ in diameter and $200 \mathrm{~mm}(L)$ in length. This workpiece is processed on a general machine tool at speeds ranging from 50 to $500 \mathrm{~m} / \mathrm{min}$. The specifications of the cutting tool are given in Table 1 .

Table 1 Data for cutting tool

\begin{tabular}{cccccc}
\hline parameters & hardness & tool lead angle & rake angle & inclination angle & nose radius \\
\hline value & $69-81 \mathrm{HRC}$ & $45^{\circ}$ & $20^{\circ}$ & $5^{\circ}$ & $R_{n}=1.2 \mathrm{~mm}$ \\
\hline
\end{tabular}

In this case, the water-based cutting fluid for the machining process is a mixture of 5\% emulsifier with $95 \%$ water by volume. The concentration $\left(\delta_{a}\right)$ of cutting fluid is 0.05 . The cutting fluid losses frequently occur during machining processes. The loss proportion is $20 \%$ (Wang et al., 2014). In this work, the initial cutting fluid volume $\left(\mathrm{v}_{0}\right)$ is $30 \mathrm{~L}$ and the additional cutting fluid volume $(\Delta \mathrm{v})$ is $6 \mathrm{~L}$. The life-cycle of the cutting fluid $\left(T_{c}\right)$ is 2 months. The density of cutting fluid or mix liquid $\left(\rho_{a}\right)$ is set to $850 \mathrm{~kg} / \mathrm{m}^{3}$ for convenient calculation. According to literature (Wang et al., 2014), the embodied energy footprint of the cutting fluid (i.e., paraffin) is $72,885 \mathrm{~kJ} / \mathrm{kg}$.

The ranges of the optimization variables (unknown values) are given in Table 2. Note that the design variable $\left(d_{s}\right)$ in the optimization procedure can be eliminated according to Eq. (38). The constant values and other relevant parameters for this optimization problem are presented in Table 3. Six multi-pass turning optimization instances were optimized by the proposed and the compared algorithms. All the experiments were carried out for six cases for each MOEA on 50 independent runs. 
Table 2 Data for the optimization variables

\begin{tabular}{ccc}
\hline variables & notation (unit) & value \\
\hline Variable bounds for roughing (optimization variables) & $d_{r L}(\mathrm{~mm})$ & 1 \\
lower bound of cutting depth & $d_{r U}(\mathrm{~mm})$ & 3 \\
upper bound of cutting depth & $f_{r L}(\mathrm{~mm} / \mathrm{rev})$ & 0.1 \\
lower bound of feed & $f_{r U}(\mathrm{~mm} / \mathrm{rev})$ & 0.9 \\
upper bound of feed & $V_{r L}(\mathrm{~m} / \mathrm{min})$ & 50 \\
lower bound of cutting speed & $V_{r U}(\mathrm{~m} / \mathrm{min})$ & 500 \\
upper bound of cutting speed & $n_{L}$ & 1 \\
lower bound of roughing passes & $n_{U}$ & 7 \\
upper bound of roughing passes & & 1 \\
Variable bounds for finishing (optimization variables) & $d_{s L}(\mathrm{~mm})$ & 3 \\
lower bound of cutting depth & $d_{s U}(\mathrm{~mm})$ & 0.1 \\
upper bound of cutting depth & $f_{S L}(\mathrm{~mm} / \mathrm{rev})$ & 0.9 \\
lower bound of feed & $f_{s U}(\mathrm{~mm} / \mathrm{rev})$ & 50 \\
upper bound of feed & $V_{s L}(\mathrm{~m} / \mathrm{min})$ & 500 \\
\hline lower bound of cutting speed & $V_{s U}(\mathrm{~m} / \mathrm{min})$ & \\
upper bound of cutting speed & & \\
\hline
\end{tabular}

Table 3 Data for the example of multi-pass turning

\begin{tabular}{|c|c|c|}
\hline Parameter & notation (unit) & value \\
\hline coefficient pertaining to idle load power & $A_{1}$ & 0.227 \\
\hline coefficient pertaining to idle load power & $A_{2}$ & $-0.667 \times 10^{-6}$ \\
\hline set of total depth of metal to be removed & $d_{t}(\mathrm{~mm})$ & {$[4,5,6,7,8,9]$} \\
\hline elastic modulus of workpiece & $E(\mathrm{MPa})$ & $2.2 \times 10^{5}$ \\
\hline maximum allowable cutting force & $F_{U}(\mathrm{~N})$ & 4903.325 \\
\hline constant pertaining to tool travel and approach time & $h_{1}(\mathrm{~min} / \mathrm{mm})$ & $7 \times 10^{-4}$ \\
\hline constant pertaining to tool travel and depart time & $h_{2}(\min )$ & 0.3 \\
\hline power when replacement tool or loading and unloading & $P_{0}(\mathrm{~kW})$ & 3.6 \\
\hline energy per insert during the production of the insert & $P_{w}(\mathrm{MJ} / \mathrm{kg})$ & 5.3 \\
\hline maximum allowable cutting power & $P_{U}(\mathrm{~kW})$ & 5 \\
\hline maximum allowable temperature & $Q_{U}\left({ }^{\circ} \mathrm{C}\right)$ & 1000 \\
\hline maximum allowable surface roughness & $R_{a}(\mu m)$ & 6.3 \\
\hline maximum stable cutting region & $S_{c}$ & 140 \\
\hline preparation time & $t_{c}(\mathrm{~min} / \mathrm{piece})$ & 1.5 \\
\hline tool exchange time & $t_{e}(\min /$ edge $)$ & 0.75 \\
\hline lower bound of tool life & $T_{L}(\min )$ & 25 \\
\hline upper bound of tool life & $T_{U}(\min )$ & 45 \\
\hline weighted coefficient of tool life & $\theta$ & 0.5 \\
\hline constant pertaining to Eqs. (11)-(12) & $p$ & 5 \\
\hline constant pertaining to Eqs. (11)-(12) & $q$ & 1.75 \\
\hline constant pertaining to Eqs. (11)-(12) & $r$ & 0.75 \\
\hline
\end{tabular}




\begin{tabular}{ccc} 
constant pertaining to Eqs. (11)-(12) & $C_{0}$ & $6 \times 10^{11}$ \\
main cutting force coefficient in Eq. (16) & $C_{F c}$ & 2650 \\
back cutting depth exponent in Eq. (16) & $x_{F c}$ & 1 \\
feed rate exponent in Eq. (16) & $y_{F c}$ & 0.75 \\
cutting speed exponent in Eq. (16) & $\eta_{F c}$ & -0.15 \\
correction coefficient in Eq. (16) & $K_{F c}$ & 0.8 \\
ratio between back force and main cutting force in Eq. (16) & $\eta_{j}$ & 0.4 \\
clamping method coefficient in Eq. (16) & $K$ & 3 \\
constant pertaining to Eqs. (22)-(23) and (30)-(31) & $\mu$ & 0.75 \\
constant pertaining to Eqs. (22)-(23) and (30)-(31) & $\vartheta$ & 0.95 \\
constant pertaining to Eqs. (22)-(23) and (30)-(31) & $k_{f}$ & 108 \\
power efficiency in Eqs. (23) and (31) & $\eta$ & 0.85 \\
constant pertaining to Eqs. (24) and (32) & $\lambda$ & 2 \\
constant pertaining to Eqs. (24) and (32) & $v$ & -1 \\
constant pertaining to Eqs. (25) and (33) & $\tau$ & 0.4 \\
constant pertaining to Eqs. (25) and (33) & $\varphi$ & 0.2 \\
constant pertaining to Eqs. (25) and (33) & $\delta$ & 0.105 \\
constant pertaining to Eqs. (25) and (33) & $k_{q}$ & 132 \\
constant pertaining to Eq. (35) & $k_{1}$ & 1 \\
constant pertaining to Eq. (36) & $k_{2}$ & 2.5 \\
constant pertaining to Eq. (37) & $k_{3}$ & 1 \\
\hline
\end{tabular}

In this experiment, the proposed MOBSA was evaluated with the six different total depths of metal to be removed: $d_{t}=4,5,6,7,8,9(\mathrm{~mm})$. That is, there are six different experiment trials. This algorithm requires the design variables (i.e., $\left.V_{r, i}, f_{r, i}, d_{r, i}, V_{s}, f_{s}, n\right)$ to solve the optimization problem and find the compromise solutions between the two challenging objectives. Due to statistic nature of the MOEAs, each experiment is carried out 50 independent runs for each MOEA on each instance. The parameters used in the proposed approach for the optimization search process were as follows:

- Population size: 100 ;

- Mix rate: 1 ;

- Number of function evaluations: 25,000 ;

In the following subsections, the performance metrics and parameter settings are described at first, and then the experimental studies are further investigated step by step.

\subsection{Performance metrics}

To measure the $P F$ obtained by the proposed algorithm, some metrics such as the Spread (Deb et al., 2002), GD, and IGD (Zitzler and Thiele, 1999) should be described as below.

(1) Spread $(\Delta)$. This metric is a diversity indicator that measures the extent of spread in the front. The metric is defined as:

$$
\Delta=\frac{\sum_{j=1}^{m} d_{j}^{e}+\sum_{i=1}^{n}\left|d_{i}-\bar{d}\right|}{\sum_{j=1}^{m} d_{j}^{e}+n \cdot \bar{d}}
$$

where $d_{i}$ denotes the Euclidean distance of each point in $P F$ to its closest point in $P F, \bar{d}$ is the average value of 
all $d_{i}, d_{j}^{e}$ is the Euclidean distance between the boundary solutions of the obtained $P F$ and the extreme solutions in the $j$-th objective from $P F^{*}, n$ is the number of $P F$ found, and $m$ is the number of objectives. If the spread is zero, then all the members of Pareto-optimal front are evenly spaced. Lower values indicate better distribution and diversity.

(2) Generational Distance (GD). The GD metric indicates how far the obtained $P F$ is from $P F^{*}$. This metric is formulated as:

$$
\mathrm{GD}=\frac{\sqrt{\sum_{i=1}^{n} d_{i}^{2}}}{n}
$$

where $n$ is the number of Pareto front points found so far, $d_{i}$ is the Euclidean distance between the $i$-th member of $P F$ obtained and the nearest member of the $P F^{*}$. A low GD value is desirable, and denotes a good convergence to $P F^{*}$.

(3) Inverse Generational Distance (IGD). This is a variant of the GD but represents a combined or comprehensive indicator. It measures the distances between each solution consisting of the optimal Pareto front and obtained front. IGD can be defined as follows:

$$
\mathrm{IGD}=\frac{\sqrt{\sum_{i=1}^{n} d_{i}^{2}}}{n}
$$

where $n$ is the number of the optimal Pareto front and $d_{i}$ is the Euclidean distance between each point of that front and the nearest member of the approximation. Fronts with a lower IGD value are desirable.

It should be noted that the true $P F^{*}$ of the considered problem may be unknown, so the non-dominated solutions found using different MOEAs for each instance in all the independent runs are regarded as $P F^{*}$ on that instance.

4.3 MOBSA versus other algorithms for turning optimization problem

To evaluate the performance of the proposed algorithm in solving the turning optimization problem, the MOBSA was compared with other classical multi-objective metaheuristics such as NSGA-II, MOPSO, MOEA/D, and MOHS. The compared MOEAs are described as follows.

- NSGA-II is one of the most popular MOEA proposed by Deb et al. (2002). The characteristic of NSGA-II is that it uses a fast non-dominated sorting and crowding distance estimation procedure. The fast non-dominated sorting technique is used to assign the parent and offspring population to different levels of non-dominated solution fronts. A crowding distance strategy is employed to maintain diversity of the population.

- MOPSO is a multi-objective particle swarm optimization algorithm proposed by Sierra and Coello Coello (2005). This proposal employs two external archives: one for storing the leaders for carrying out the flight and the other one for storing the final solutions. The crowding factor is used to remove the list of leaders whenever the external archive is full of final solutions. Only the leaders with the best crowding values are maintained.

- MOEA/D is invented by Zhang et al. (2007). It decomposes a multi-objective problem into a number of scalar sub-problems and optimizes them simultaneously. Since MOEA/D is used to deal with constrained multi-objective problem in this work, a constraint handling mechanism is incorporated into the original MOEA/D called cMOEA/D (Zapotecas Martinez and Coello Coello, 2014).

- MOHS is a general version of multi-objective harmony search algorithm. Each new generated solution is based on three rules: (a) harmony memory consideration, (b) pitch adjustment and (c) random selection. 
MOHS has been applied successfully into many optimization fields.

It is well known that the parameter settings can affect the behavior of the algorithm. Thus, pilot experiments were conducted to find an appropriate parameter configuration. The parameter settings are determined through performing a considerable number of experiments. The most parameter values are fixed. However, some parameters are related to the particular problem. For NSGA-II, the simulated binary crossover (SBX) and polynomial mutation are used. The distribution indexes in both SBX and the polynomial mutation are set to 20 . The crossover rate is 0.9 and mutation rate is 0.1 . For MOPSO, the mutation rate is set to 0.2 and perturbation index is set to 0.1 . For cMOEA/D, the neighborhood size is set to 20. For MOHS, harmony memory consideration rate (HMCR) is set to 0.9 and pitch adjustment rate (PAR) is set to 0.1 . Band width (BW) is set to 0.05 . The other suggested parameter setting of the original authors for each algorithm was adopted and each experiment was conducted 50 independent runs for each algorithm on each instance. To make a fair comparison, the stop condition is set to 25,000 function evaluations for each algorithm. The population size is set to 100 for NSGA-II, cMOEA/D, MOPSO and MOBSA. The population size is set to 40 for MOHS. In addition, the external archive size is set to 50. Owing to their stochastic nature, the following statistical analysis was necessary to give confidential comparisons. Firstly, the kolmogorov-smirnov test was performed to determine whether the result has a normal distribution. If the results were not normally distributed, the t-test was used to compare the performance of each algorithm; otherwise, a wilcoxon rank sum test was applied to the mean values. Two statistical tests were executed on the pairs of the algorithms to measure the significant differences between the results obtained by the different algorithms. The confidence level for all the tests was set to 95\% (corresponding to a $\alpha=0.05$ ). The symbols "+," "-,", and "=" indicate that the proposed MOBSA algorithm is significantly better than, worse than, and similar to that of its first or second best counterpart on average in terms of each metric, respectively.

Table 4 presents the statistical results of GD, $\Delta$, and IGD. The optimal metric results are highlighted with bold font. It can be clearly observed from Table 4 that the proposed MOBSA outperforms its counterparts when solving the multi-pass turning optimization problem. Especially for the comprehensive metric IGD and convergence metric GD, the performance of the proposed algorithm is overwhelming without exception. Fig. 4 shows the $P F$ approximations found in the run with the best IGD value of each metaheuristic for each instance. It is evident that the MOBSA can obtain better approximations than its rivals with regard to the convergence and spread metrics. As depicted in Fig. 4, the MOBSA can converge closer to the $P F^{*}$ (i.e., reference points) of the problems, while the other algorithms cannot converge to the optimal $P F^{*}$ except for the problem one $\left(d_{t}=4\right)$ under the same number of function evaluations. Fig. 4 also gives an obvious illustration that the Pareto front obtained by the MOBSA has a wider and more uniform spread. The superior performance of the MOBSA can be attributed to the dual population. The MOBSA with a dual population has a greater chance to search for promising areas, which improves the exploration ability. The statistical results of the IGD metric over 50 independent runs are plotted as boxplots in Fig. 5, with the small square representing the average IGD value obtained by each algorithm. The vertical axis of each subfigure indicates the IGD value and the horizontal axis denotes the corresponding algorithms. These boxplots present an intuitive distribution tendency of the statistical results over 50 runs. From Fig. 5, it is clear that the box shape of the MOBSA is narrower than that of the other metaheuristics on most problems. This shows that the MOBSA has a more stable performance in comparison with four other approaches. The box position of the MOBSA is also lower than that of its compared approaches, which implies that the MOBSA is superior to the other algorithms in terms of the IGD metric. Moreover, it is consistent with the results from Table 4. Therefore, it can be concluded that the MOBSA has been successfully applied to the multi-pass turning operations and outperforms the other MOEAs considered in this study. 
Table 4 Performance comparisons among MOBSA and other algorithms considered on each instance

\begin{tabular}{|c|c|c|c|c|c|c|}
\hline$d_{t}$ & Metrics & $\begin{array}{c}\text { NSGA-II } \\
\text { average(std) }\end{array}$ & $\begin{array}{c}\text { MOPSO } \\
\text { average(std) }\end{array}$ & $\begin{array}{c}\text { MOHS } \\
\text { average(std) }\end{array}$ & $\begin{array}{l}\text { cMOEA/D } \\
\text { average(std) }\end{array}$ & $\begin{array}{c}\text { MOBSA } \\
\text { average(std) }\end{array}$ \\
\hline \multirow{3}{*}{4} & $\Delta$ & $5.34 \mathrm{e}-01(5.4 \mathrm{e}-02)$ & $4.93 \mathrm{e}-01(1.0 \mathrm{e}-01)$ & $8.94 \mathrm{e}-01(1.3 \mathrm{e}-01)$ & $5.62 \mathrm{e}-01(6.9 \mathrm{e}-02)$ & $3.87 e-01(4.8 e-02)+$ \\
\hline & GD & $1.70 \mathrm{e}-03(5.6 \mathrm{e}-04)$ & $2.09 \mathrm{e}-03(1.2 \mathrm{e}-03)$ & $1.22 \mathrm{e}-01(9.3 \mathrm{e}-02)$ & $6.77 \mathrm{e}-04(3.9 \mathrm{e}-04)$ & $4.05 e-04(4.4 e-04)+$ \\
\hline & IGD & $8.39 \mathrm{e}-04(4.1 \mathrm{e}-04)$ & $9.48 \mathrm{e}-04(2.9 \mathrm{e}-04)$ & $2.29 \mathrm{e}-02(1.3 \mathrm{e}-02)$ & $6.61 \mathrm{e}-04(3.9 \mathrm{e}-04)$ & $6.12 e-04(1.8 e-04)+$ \\
\hline \multirow{3}{*}{5} & $\Delta$ & $7.58 \mathrm{e}-01(5.2 \mathrm{e}-02)$ & $6.60 \mathrm{e}-01(3.4 \mathrm{e}-02)$ & $9.46 e-01(4.7 e-02)$ & $7.99 \mathrm{e}-01(8.1 \mathrm{e}-02)$ & $6.55 e-01(2.8 e-02)+$ \\
\hline & GD & $4.46 \mathrm{e}-03(1.2 \mathrm{e}-03)$ & $4.08 \mathrm{e}-03(1.0 \mathrm{e}-03)$ & $4.99 \mathrm{e}-02(3.5 \mathrm{e}-02)$ & $5.70 \mathrm{e}-03(6.6 \mathrm{e}-03)$ & $3.55 e-03(7.1 e-04)+$ \\
\hline & IGD & $4.51 \mathrm{e}-03(2.8 \mathrm{e}-04)$ & $4.40 \mathrm{e}-03(5.2 \mathrm{e}-04)$ & $1.84 \mathrm{e}-02(8.5 \mathrm{e}-03)$ & $6.58 \mathrm{e}-03(4.6 \mathrm{e}-03)$ & $4.30 \mathrm{e}-03(5.0 \mathrm{e}-04)+$ \\
\hline \multirow{3}{*}{6} & $\Delta$ & $8.56 \mathrm{e}-01(4.6 \mathrm{e}-02)$ & $7.41 e-01(8.7 e-02)$ & $9.55 \mathrm{e}-01(5.7 \mathrm{e}-02)$ & $7.58 \mathrm{e}-01(1.3 \mathrm{e}-01)$ & $7.83 \mathrm{e}-01(4.7 \mathrm{e}-02)-$ \\
\hline & GD & $5.56 \mathrm{e}-03(2.6 \mathrm{e}-03)$ & $5.79 \mathrm{e}-03(4.7 \mathrm{e}-03)$ & $1.12 \mathrm{e}-01(1.3 \mathrm{e}-01)$ & $3.66 \mathrm{e}-03(8.6 \mathrm{e}-03)$ & $3.40 e-03(2.1 e-03)+$ \\
\hline & IGD & $5.43 e-03(4.3 e-03)$ & $5.33 \mathrm{e}-03(2.0 \mathrm{e}-03)$ & $3.44 \mathrm{e}-02(2.8 \mathrm{e}-02)$ & $5.93 \mathrm{e}-03(7.4 \mathrm{e}-03)$ & $2.78 \mathrm{e}-03(3.0 \mathrm{e}-03)+$ \\
\hline \multirow{3}{*}{7} & $\Delta$ & $8.79 e-01(4.6 e-02)$ & $7.92 \mathrm{e}-01(7.1 \mathrm{e}-02)$ & $9.40 \mathrm{e}-01(6.6 \mathrm{e}-02)$ & $7.71 \mathrm{e}-01(1.1 \mathrm{e}-01)$ & $7.96 \mathrm{e}-01(3.2 \mathrm{e}-02)-$ \\
\hline & GD & $3.15 \mathrm{e}-03(2.2 \mathrm{e}-03)$ & $4.47 \mathrm{e}-03(3.9 \mathrm{e}-03)$ & $6.45 \mathrm{e}-02(8.5 \mathrm{e}-02)$ & $2.34 \mathrm{e}-03(3.2 \mathrm{e}-03)$ & $7.46 e-04(8.1 e-04)+$ \\
\hline & IGD & $4.72 \mathrm{e}-03(2.5 \mathrm{e}-03)$ & $6.91 \mathrm{e}-03(1.7 \mathrm{e}-03)$ & $2.11 \mathrm{e}-02(1.4 \mathrm{e}-02)$ & $5.18 \mathrm{e}-03(4.2 \mathrm{e}-03)$ & $3.81 e-03(2.6 e-03)+$ \\
\hline \multirow{3}{*}{8} & $\Delta$ & $9.24 \mathrm{e}-01(2.3 \mathrm{e}-02)$ & $8.90 e-01(4.3 e-02)$ & $9.78 \mathrm{e}-01(2.6 \mathrm{e}-02)$ & $9.22 \mathrm{e}-01(5.5 \mathrm{e}-02)$ & $9.15 \mathrm{e}-01(2.6 \mathrm{e}-02)-$ \\
\hline & GD & $4.49 \mathrm{e}-03(1.4 \mathrm{e}-03)$ & $8.34 \mathrm{e}-03(2.7 \mathrm{e}-03)$ & $5.18 \mathrm{e}-02(9.4 \mathrm{e}-02)$ & $4.54 \mathrm{e}-03(6.9 \mathrm{e}-03)$ & $2.75 e-03(1.9 e-03)+$ \\
\hline & IGD & $1.07 \mathrm{e}-02(1.6 \mathrm{e}-03)$ & $1.10 \mathrm{e}-02(1.3 \mathrm{e}-03)$ & $2.11 \mathrm{e}-02(7.9 \mathrm{e}-03)$ & $1.55 e-02(7.5 e-03)$ & $1.03 e-02(2.7 e-03)+$ \\
\hline \multirow{3}{*}{9} & $\Delta$ & $8.98 \mathrm{e}-01(4.4 \mathrm{e}-02)$ & $8.37 e-01(8.2 e-02)$ & $9.61 e-01(4.3 e-02)$ & $8.98 \mathrm{e}-01(9.3 \mathrm{e}-02)$ & $8.93 \mathrm{e}-01(4.0 \mathrm{e}-02)-$ \\
\hline & GD & $7.76 \mathrm{e}-03(2.1 \mathrm{e}-03)$ & $6.17 \mathrm{e}-03(3.1 \mathrm{e}-03)$ & $6.98 \mathrm{e}-02(1.0 \mathrm{e}-01)$ & $1.23 \mathrm{e}-02(4.4 \mathrm{e}-03)$ & $5.24 \mathrm{e}-03(6.0 \mathrm{e}-03)+$ \\
\hline & IGD & $1.04 \mathrm{e}-02(1.9 \mathrm{e}-03)$ & $9.51 \mathrm{e}-03(6.2 \mathrm{e}-03)$ & $2.73 \mathrm{e}-02(1.2 \mathrm{e}-02)$ & $1.39 \mathrm{e}-02(6.7 \mathrm{e}-03)$ & $9.19 e-03(2.8 e-03)=$ \\
\hline
\end{tabular}

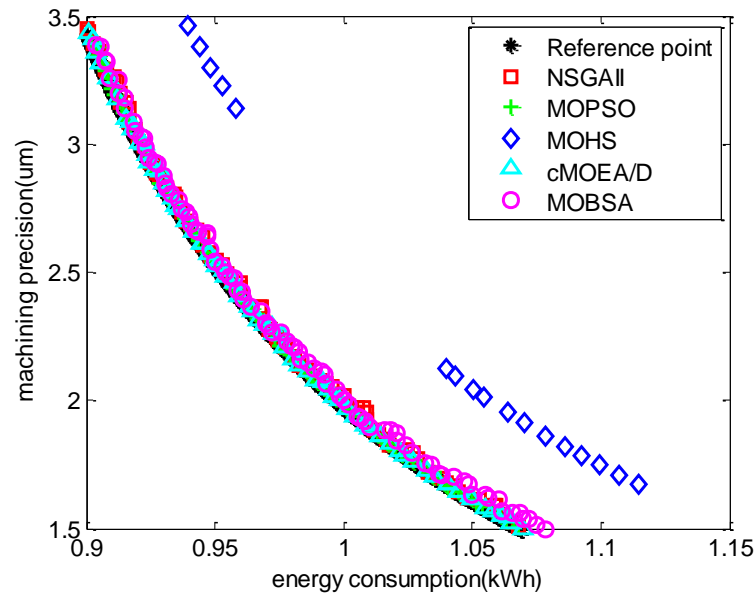

(a)

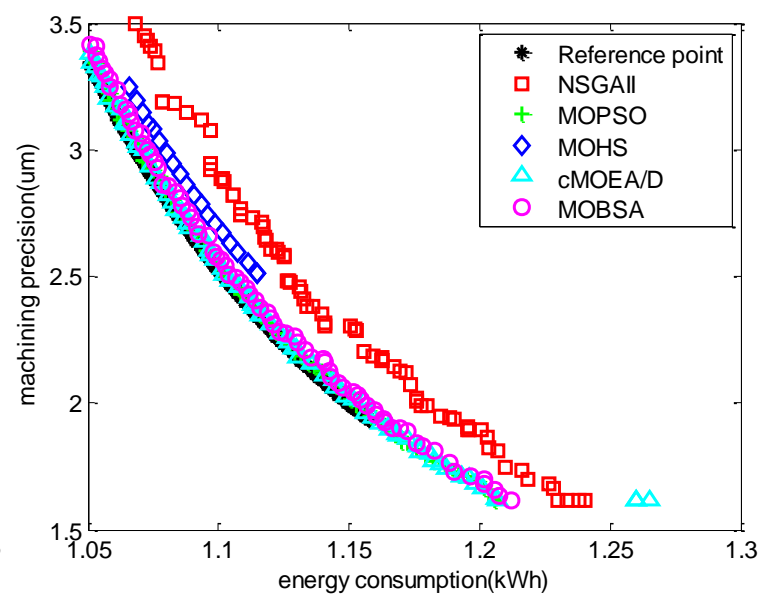

(b) 


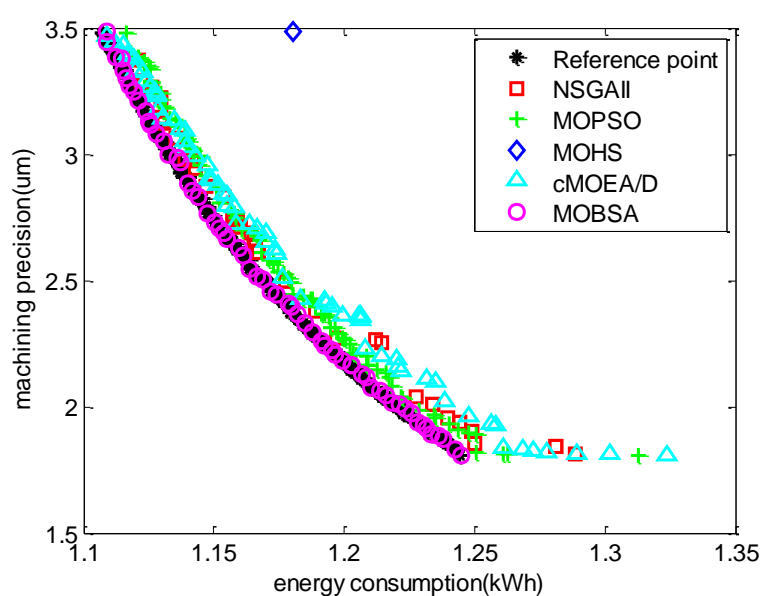

(c)

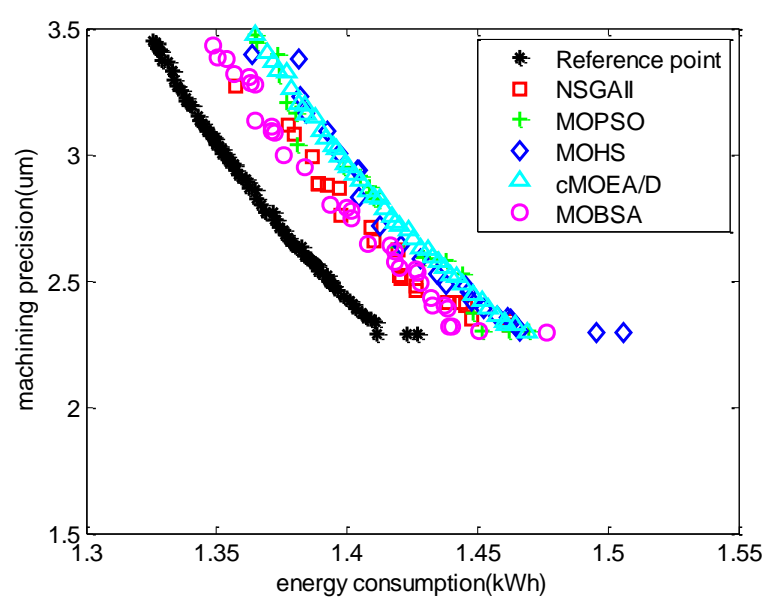

(e)

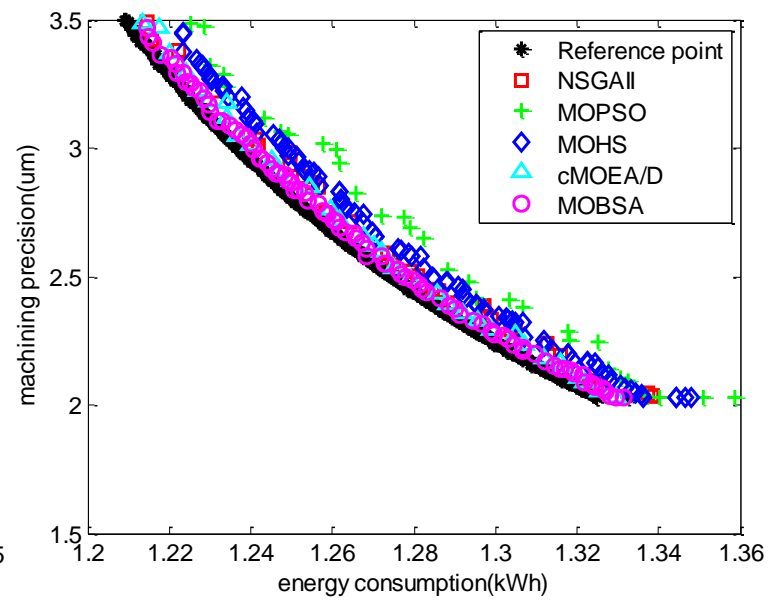

(d)

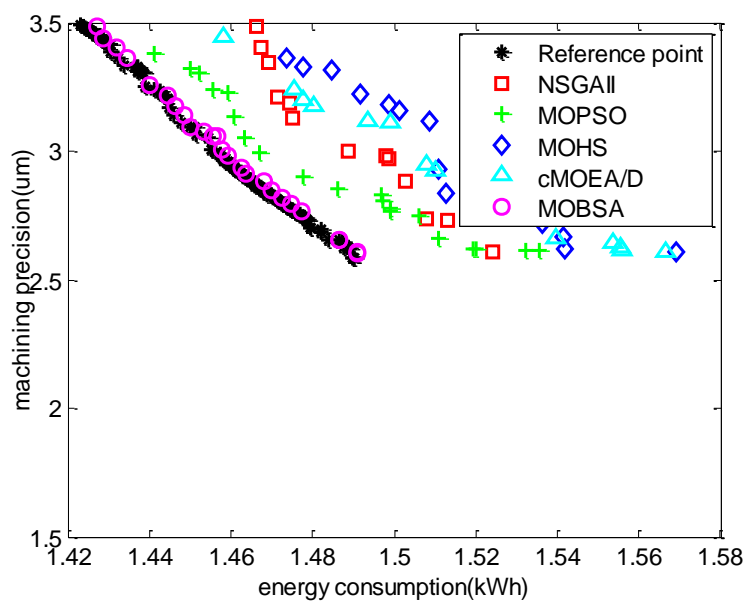

(f)

Fig. 4 Pareto front approximations with the best IGD metric by different multi-objective algorithms on different instances (a) $d_{t}=4$, (b) $d_{t}=5$, (c) $d_{t}=6$, (d) $d_{t}=7$, (e) $d_{t}=8$, (f) $d_{t}=9$

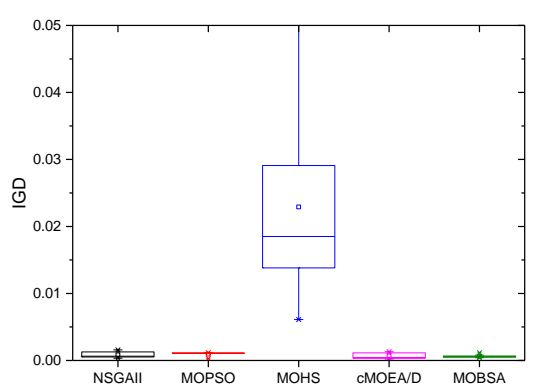

(a)

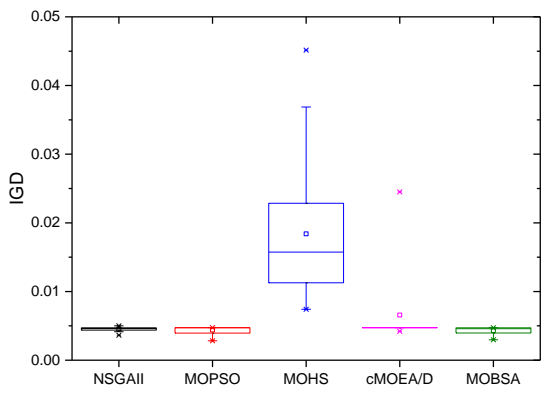

(b)

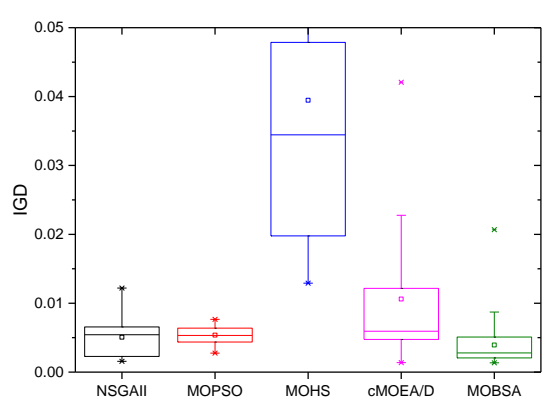

(c) 


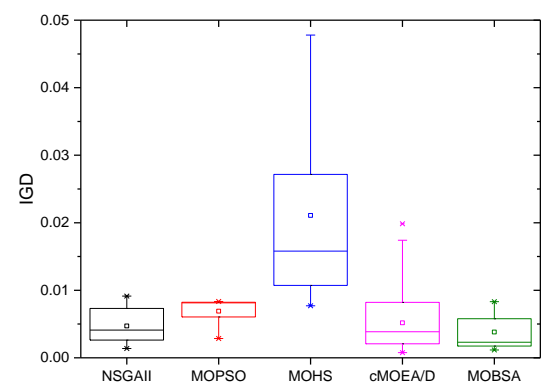

(d)

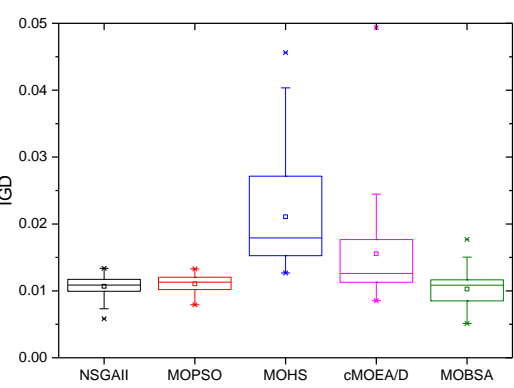

(e)

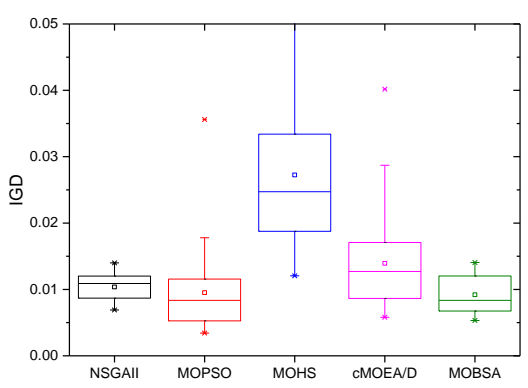

(f)

Fig. 5 Boxplot study on IGD metric for each instance (a) $d_{t}=4$, (b) $d_{t}=5$, (c) $d_{t}=6$, (d) $d_{t}=7$, (e) $d_{t}=8$, (f) $d_{t}=9$

Due to the conflicting nature of multiple criteria, the results of the MOP for minimum energy consumption and machining precision reveal that higher energy consumption accompanies superior machining precision and vice versa. Because the non-dominated solutions contain many points, a good Pareto front should be composed of a limited number of points that are as close as possible to the $P F^{*}$ and uniformly spread as well. Fig. 6 shows the trade-offs obtained by MOBSA algorithm for the multi-objective optimization. It can be observed from Fig. 6 that the minimum energy consumption is observed at design point $\mathrm{A}$ where the machining precision is lowest for different values of the parameter $d_{t}$. On the other hand, the maximum energy consumption occurs at design point $\mathrm{C}$ where the machining precision is highest. Specifications of three sample design points A-C in the Pareto optimal fronts for different values of $d_{t}$ are summarized in Table 5. It is observed from Fig. 6 and Table 5 that the energy consumption increases with the increase of $d_{t}$. These observations further verify our views that the two objectives of the optimization problem are conflicting. According to Table 5, more than $12 \%$ energy can be saved by the developed algorithm with parameters of point A compared to those of point $\mathrm{C}$. In addition, decision-maker can adopt TOPSIS method (Gwo-Hshiung, 2010) to select the best solution from the non-dominate solution. The detailed process of the TOPSIS is not considered in this paper due to space limitations.

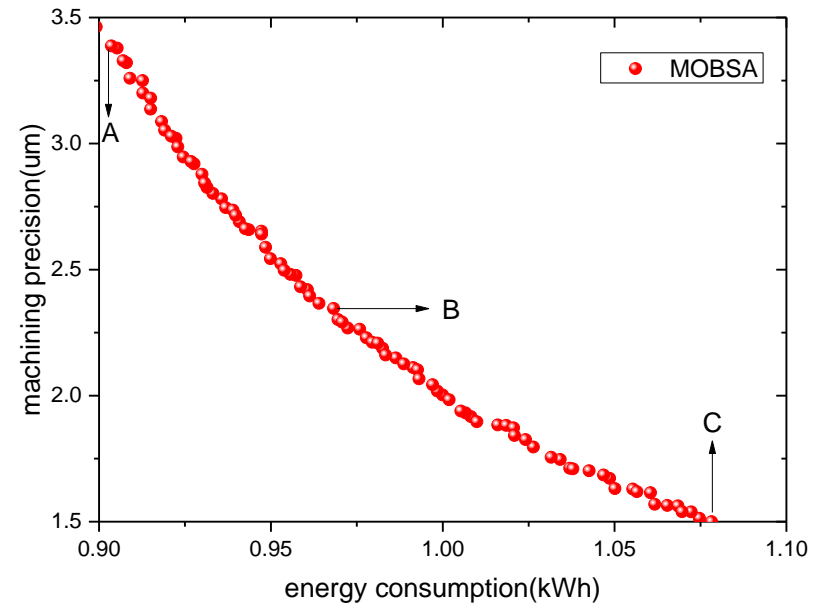

(a)

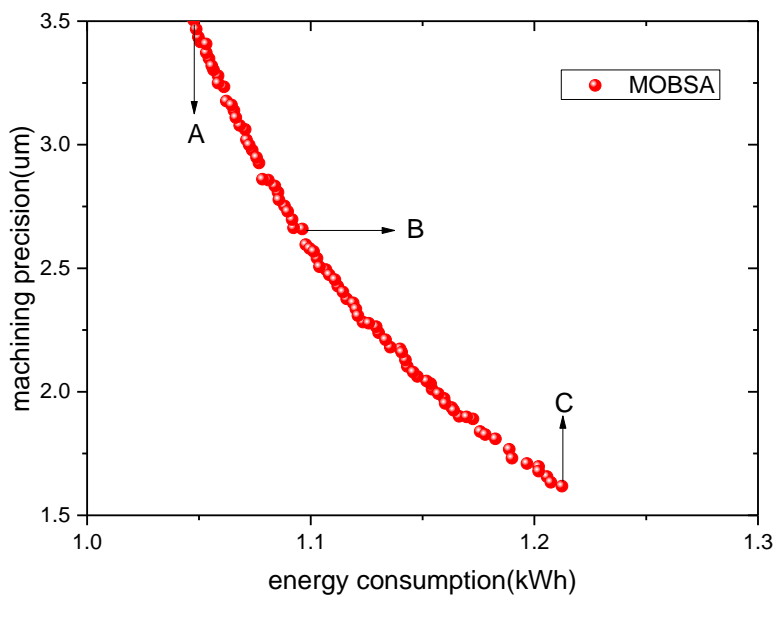

(b) 


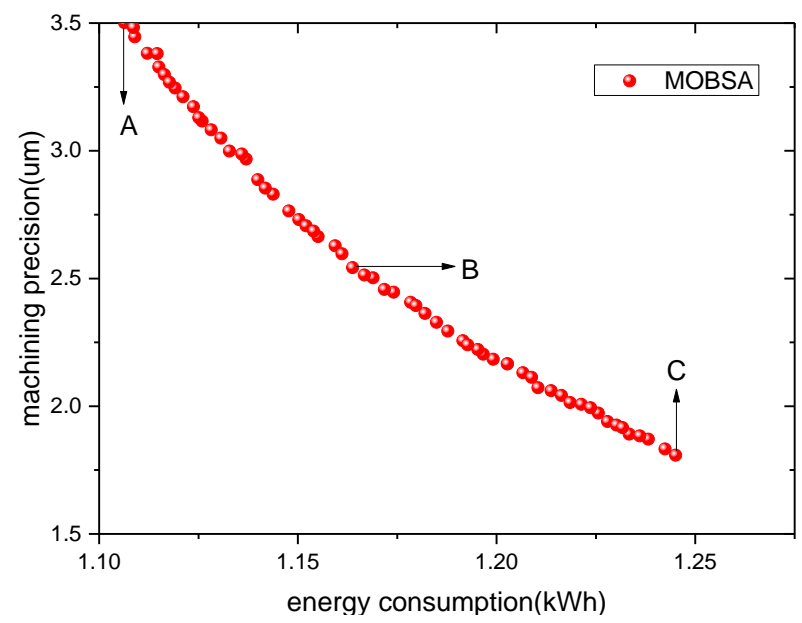

(c)

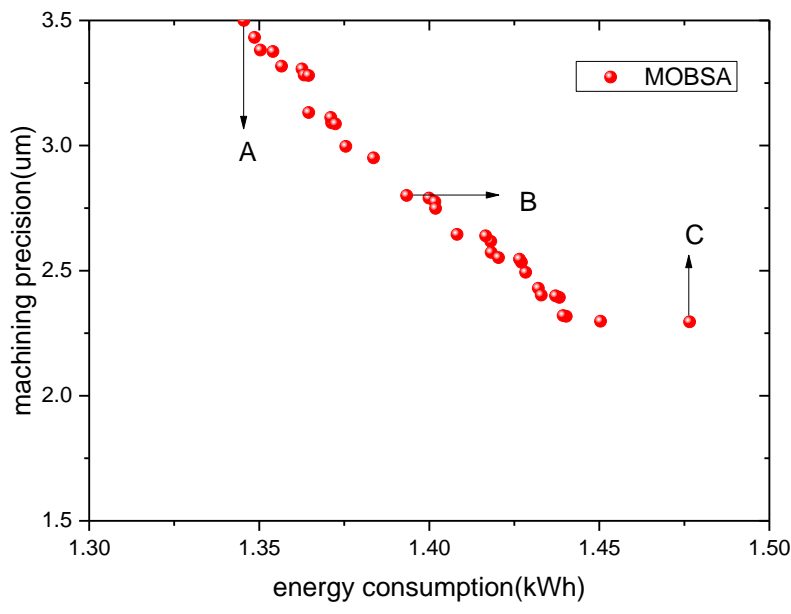

(e)

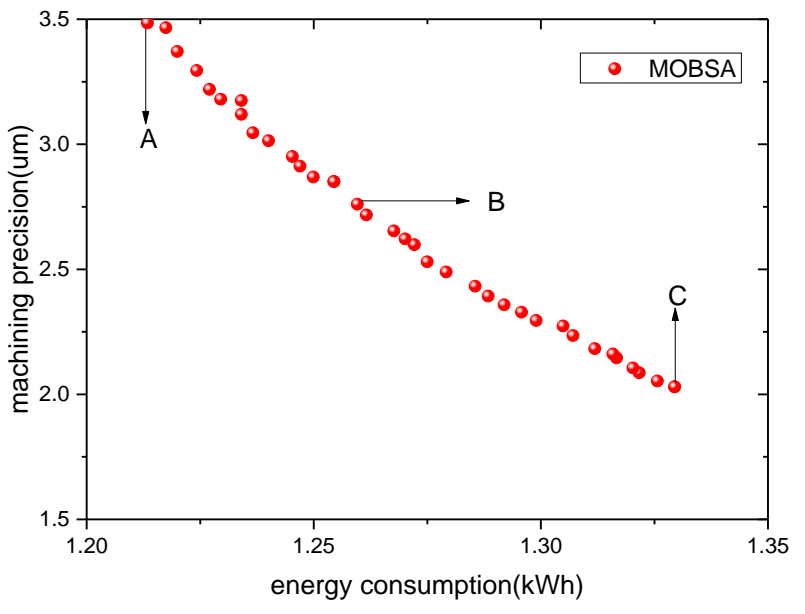

(d)

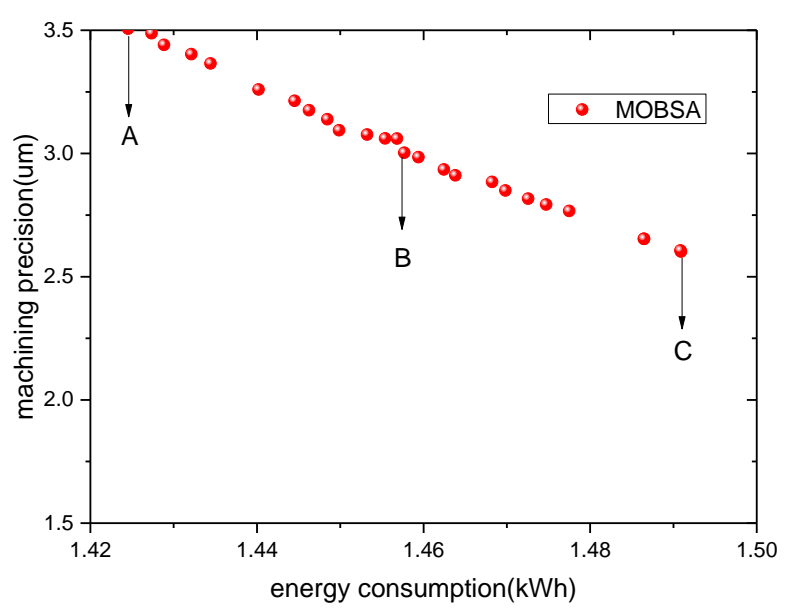

(f)

Fig. 6 The distribution of Pareto front for energy consumption and machining precision using MOBSA algorithm (a) $d_{t}=4$, (b) $d_{t}=5$, (c) $d_{t}=6$, (d) $d_{t}=7$, (e) $d_{t}=8$, (f) $d_{t}=9$

Table 5 Specifications of three sample design points A-C in Pareto optimal fronts for different values of $d_{t}$.

\begin{tabular}{|c|c|c|c|c|c|c|c|c|c|c|c|c|c|c|}
\hline \multirow{2}{*}{ case } & \multirow{2}{*}{ point } & \multicolumn{11}{|c|}{ design variables } & \multirow{2}{*}{$f_{1}(\mathrm{kWh})$} & \multirow{2}{*}{$f_{2}(\mu \mathrm{m})$} \\
\hline & & $V_{r, 1}$ & $V_{r, 2}$ & $V_{r, 3}$ & $f_{r, 1}$ & $f_{r, 2}$ & $f_{r, 3}$ & $d_{r, 1}$ & $d_{r, 2}$ & $d_{r, 3}$ & $V_{s}$ & $f_{s}$ & & \\
\hline \multirow{3}{*}{$d_{t}=4$} & A & 100.25 & - & - & 0.74 & - & - & 3.0 & - & - & 181.94 & 0.29 & 0.904 & 3.387 \\
\hline & B & 100.61 & - & - & 0.73 & - & - & 3.0 & - & - & 212.10 & 0.18 & 0.968 & 2.346 \\
\hline & $\mathrm{C}$ & 101.03 & - & - & 0.73 & - & - & 3.0 & - & - & 255.53 & 0.10 & 1.078 & 1.500 \\
\hline \multirow{3}{*}{$d_{t}=5$} & A & 119.05 & 112.87 & - & 0.90 & 0.90 & - & 2.07 & 1.92 & - & 184.36 & 0.26 & 1.049 & 3.468 \\
\hline & B & 119.45 & 113.51 & - & 0.90 & 0.90 & - & 1.99 & 2.01 & - & 205.98 & 0.18 & 1.096 & 2.659 \\
\hline & $\mathrm{C}$ & 119.95 & 113.77 & - & 0.86 & 0.90 & - & 2.06 & 1.94 & - & 264.19 & 0.10 & 1.212 & 1.618 \\
\hline \multirow{3}{*}{$d_{t}=6$} & A & 121.13 & 113.35 & - & 0.70 & 0.82 & - & 2.57 & 2.42 & - & 199.71 & 0.22 & 1.109 & 3.482 \\
\hline & B & 121.08 & 113.31 & - & 0.70 & 0.82 & - & 2.57 & 2.43 & - & 229.65 & 0.15 & 1.164 & 2.542 \\
\hline & $\mathrm{C}$ & 121.23 & 113.43 & - & 0.69 & 0.81 & - & 2.57 & 2.42 & - & 266.32 & 0.10 & 1.245 & 1.808 \\
\hline \multirow{2}{*}{$d_{t}=7$} & A & 135.72 & 125.54 & - & 0.49 & 0.55 & - & 3.0 & 3.0 & - & 198.55 & 0.19 & 1.213 & 3.485 \\
\hline & B & 136.02 & 125.82 & - & 0.49 & 0.54 & - & 3.0 & 3.0 & - & 226.83 & 0.15 & 1.260 & 2.761 \\
\hline
\end{tabular}




\begin{tabular}{|c|c|c|c|c|c|c|c|c|c|c|c|c|c|c|}
\hline & $\mathrm{C}$ & 136.10 & 125.89 & - & 0.49 & 0.54 & - & 3.0 & 3.0 & - & 265.64 & 0.10 & 1.329 & 2.030 \\
\hline \multirow{3}{*}{$d_{t}=8$} & A & 147.08 & 138.56 & 130.55 & 0.59 & 0.71 & 0.63 & 2.32 & 2.18 & 2.49 & 221.14 & 0.17 & 1.345 & 3.500 \\
\hline & B & 146.20 & 137.71 & 130.04 & 0.58 & 0.69 & 0.63 & 2.32 & 2.10 & 2.58 & 225.76 & 0.13 & 1.393 & 2.800 \\
\hline & $\mathrm{C}$ & 152.46 & 143.16 & 135.52 & 0.44 & 0.67 & 0.57 & 2.44 & 2.00 & 2.56 & 259.86 & 0.10 & 1.477 & 2.295 \\
\hline \multirow{3}{*}{$d_{t}=9$} & A & 152.85 & 143.07 & 132.01 & 0.51 & 0.48 & 0.63 & 2.56 & 2.89 & 2.54 & 228.06 & 0.14 & 1.427 & 3.488 \\
\hline & B & 152.95 & 143.20 & 132.11 & 0.51 & 0.48 & 0.63 & 2.55 & 2.90 & 2.55 & 241.56 & 0.12 & 1.458 & 3.003 \\
\hline & $\mathrm{C}$ & 152.63 & 142.86 & 131.82 & 0.51 & 0.48 & 0.63 & 2.56 & 2.89 & 2.54 & 262.94 & 0.10 & 1.491 & 2.606 \\
\hline
\end{tabular}

\subsection{Sensitivity analysis}

To identify the effect of the design variables on the energy consumption and machining precision, design point B for one instance in Fig. 6 (a) is investigated in this subsection. The design variable vector is $\left(V_{r, i}, f_{r, i}, d_{r, i}, V_{s}, f_{s}, n\right)$, where the $n$ is equal to 1 in point $\mathrm{B}$. Here, the four design variables $V_{r}, f_{r}, V_{s}$ and $f_{s}$ were chosen as control parameters in this optimization problem, while the other parameters were fixed at the value for point $\mathrm{B}$.

To study how sensitive the MOBSA is to the four parameters for multi-pass turning parameter optimization, this work attempted to cover a feasible range of values for each control parameter. Four different values were considered for $V_{r} \in[100.61,100.62,100.63,100.64] \mathrm{m} / \mathrm{min}$, four different variable values for $f_{r} \in$ $[0.73,0.74,0.75,0.76] \mathrm{mm} / \mathrm{rev}$, four variable values for $V_{s} \in[212.10,213.10,214.10,215.10] \mathrm{m} / \mathrm{min}$, and four values for $f_{s} \in[0.18,0.19,0.20,0.21] \mathrm{mm} / \mathrm{rev}$. Note that these control parameters varied near point B in Fig. 6 (a) because they needed to satisfy all constraints. This experiment took $V_{r}$ and $f_{r}$ as a configuration and measured all 16 different parameter combinations as shown in Fig. 7. Similarly, $V_{s}$ and $f_{s}$ were considered as another parameter configuration. The experiment tested 16 different parameter combinations on this instance as presented in Fig. 8. Fig. 7 and Fig. 8 reveal the effect of parameters on the energy consumption $\left(f_{1}\right)$ and machining accuracy $\left(f_{2}\right)$ when the two conflicting bi-objectives are separated. It can be observed from Fig. 7 that different parameter settings have little effect on the two criteria. This implies that the parameters $V_{r}$ and $f_{r}$ are not sensitive to the objective $f_{1}$ and $f_{2}$. From Fig. 8, one can observe that different combinations can lead to different performances on this optimization problem. Additionally, one interesting observation from Fig. 8 is that when the parameters $V_{\mathrm{s}}$ and $f_{\mathrm{s}}$ can improve one objective, it will result in the deterioration of the other objective.

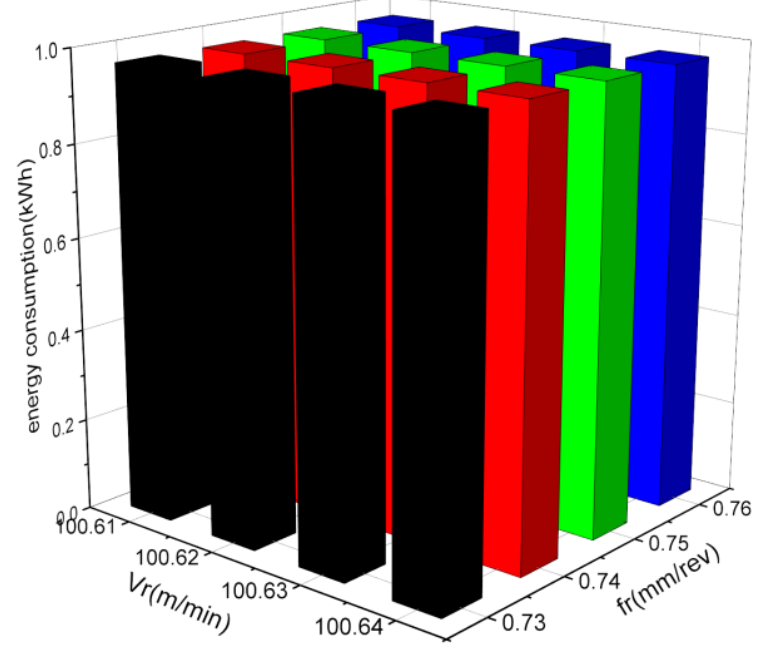

(a)

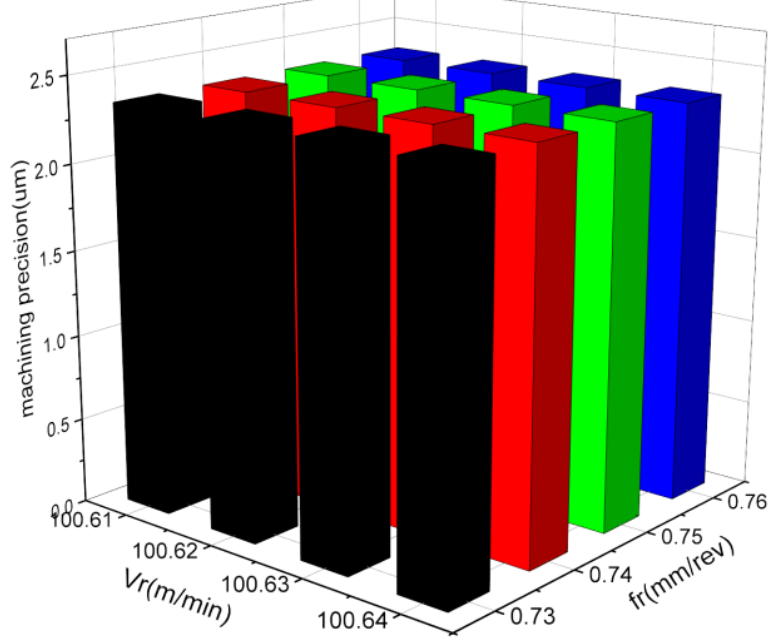

(b) 
Fig. 7. The energy consumption and machining precision using MOBSA algorithm with 16 different combinations of $V_{\mathrm{r}}$ and $f_{\mathrm{r}}$ on point $\mathrm{B}$ for instance one (a) energy consumption, (b) machining precision

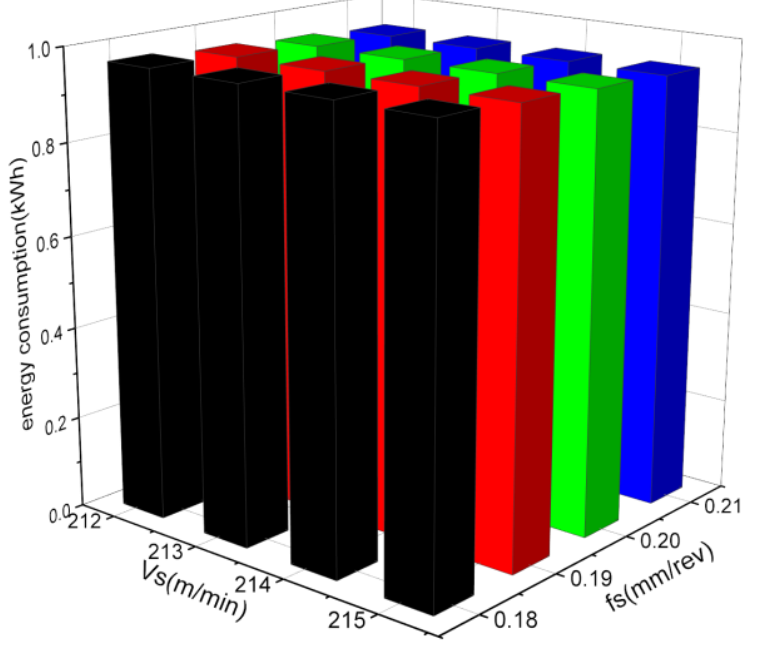

(a)

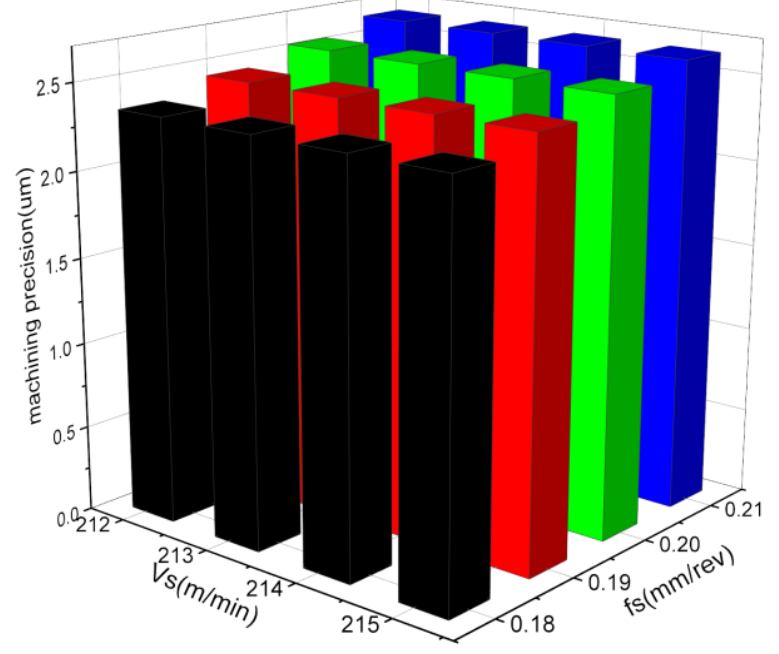

(b)

Fig. 8. The energy consumption and machining precision using MOBSA algorithm with 25 different combinations of $V_{\mathrm{s}}$ and $f_{\mathrm{s}}$ on point $\mathrm{B}$ for instance one (a) energy consumption, (b) machining precision

\section{Conclusions and future work}

Reducing energy and improving machining quality are highly important in machining processes. The primary objective of machining processes is to improve production or quality efficiency. However, sustainable manufacturing has attracted increasingly more attention with the advent of climate change and environmental deterioration. This paper studies a multi-pass turning operation process, which is an important step in most manufacturing processes. This paper first formulates a multi-objective multi-pass turning operation model considering quality and energy efficiency. The energy consumption criterion consists of energy consumption for machining, machine idle, tool replacement, tool wear, and the cutting fluid. To obtain the trade-offs between energy consumption and cutting quality, this current study presents a multi-objective backtracking search algorithm, which is used to solve the cutting parameters in a multi-pass turning process. The proposed MOBSA is compared with NSGA-II, MOPSO, cMOEA/D, and MOHS for different parameter optimization problems. The experimental results reveal that the MOBSA outperforms its rivals on this problem. Furthermore, the parameter sensitivity on the considered instances has been investigated.

With respect to future work, an interesting issue that deserves further study is the extension of MOBSA to other machining processes, such as milling and grinding. This optimization method can also be applied to scheduling problems with energy consumption, such as energy-efficient dynamic job shop scheduling problem.

\section{Acknowledgments}

This research work was supported by the National Science Foundation of China (NSFC) under Grant no. 51435009 and 51375004, special projects financed by postdoctoral Science Foundation of China under Grant no. 2015T80798, the Youth Science \& Technology Chenguang Program of Wuhan under Grant no. 2015070404010187 and the Fundamental Research Funds for the Central Universities, HUST: Grant no. 2015 TS061. 


\section{References}

An, L., 2003. Optimization of machining parameters in multi-pass turning and milling operations. Concordia University. Arif, M., Stroud, I.A., Akten, O., 2013. A model to determine the optimal parameters for sustainable-energy machining in a multi-pass turning operation. Proceedings of the Institution of Mechanical Engineers, Part B: Journal of Engineering Manufacture, 0954405413508945.

Askarzadeh, A., Coelho, L.D., 2014. A backtracking search algorithm combined with Burger's chaotic map for parameter estimation of PEMFC electrochemical model. International Journal of Hydrogen Energy 39, 11165-11174.

Basar, O., Mine, I., Secil, E., 2013. Multi-Criteria Decision Aid for Sustainable Energy Prioritization Using Fuzzy Axiomatic Design. International Journal of Energy Optimization and Engineering (IJEOE) 2, 1-20.

Brundtland, G., Khalid, M., Agnelli, S., Al-Athel, S., Chidzero, B., Fadika, L., Hauff, V., Lang, I., Shijun, M., de Botero, M.M., 1987. Our Common Future (''Brundtland report \').

Camposeco-Negrete, C., 2013. Optimization of cutting parameters for minimizing energy consumption in turning of AISI 6061 T6 using Taguchi methodology and ANOVA. Journal of Cleaner Production 53, 195-203.

Chauhan, P., Pant, M., Deep, K., 2015. Parameter optimization of multi-pass turning using chaotic PSO. International Journal of Machine Learning and Cybernetics 6, 319-337.

Chen, M.C., 2004. Optimizing machining economics models of turning operations using the scatter search approach. Int. J. Prod. Res. 42, 2611-2625.

Chen, M.C., Tsai, D.M., 1996. A simulated annealing approach for optimization of multi-pass turning operations. Int. J. Prod. Res. 34, 2803-2825.

Civicioglu, P., 2013. Backtracking Search Optimization Algorithm for numerical optimization problems. Appl Math Comput 219, 8121-8144.

Dai, M., Tang, D., Giret, A., Salido, M.A., Li, W.D., 2013. Energy-efficient scheduling for a flexible flow shop using an improved genetic-simulated annealing algorithm. Robotics and Computer-Integrated Manufacturing 29, 418-429.

Deb, K., Pratap, A., Agarwal, S., Meyarivan, T., 2002. A fast and elitist multiobjective genetic algorithm: NSGA-II. Evolutionary Computation, IEEE Transactions on 6, 182-197.

El-Fergany, A., 2015. Optimal allocation of multi-type distributed generators using backtracking search optimization algorithm. Int. J. Electr. Power Energy Syst. 64, 1197-1205.

Fang, K., Uhan, N., Zhao, F., Sutherland, J.W., 2011. A new approach to scheduling in manufacturing for power consumption and carbon footprint reduction. J Manuf Syst 30, 234-240.

Ganesan, T., Vasant, P., Elamvazuthi, I., 2013. Normal-boundary intersection based parametric multi-objective optimization of green sand mould system. J Manuf Syst 32, 197-205.

Gao, L., Huang, J.D., Li, X.Y., 2012. An effective cellular particle swarm optimization for parameters optimization of a multi-pass milling process. Appl. Soft. Comput. 12, 3490-3499.

Guo, Y., Duflou, J.R., Qian, J., Tang, H., Lauwers, B., 2015. An operation-mode based simulation approach to enhance the energy conservation of machine tools. Journal of Cleaner Production 101, 348-359.

Gwo-Hshiung, T., 2010. Multiple attribute decision making: methods and applications. Multiple Attribute Decision Making: Methods and Applications.

Kara, S., Manmek, S., Herrmann, C., 2010. Global manufacturing and the embodied energy of products. CIRP Annals Manufacturing Technology 59, 29-32.

Li, C., Cui, L., Liu, F., Li, L., 2013. Multi-objective NC Machining Parameters Optimization Model for High Efficiency and Low Carbon. Chinese Journal of Mechanical Engineering 49, 87-96.

Li, Y.Z., Li, X.P., Gupta, J.N.D., 2015. Solving the multi-objective flowline manufacturing cell scheduling problem by hybrid harmony search. Expert Syst. Appl. 42, 1409-1417. 
Lin, W., Yu, D.Y., Zhang, C., Liu, X., Zhang, S., Tian, Y., Liu, S., Xie, Z., 2015. A multi-objective teaching-learning-based optimization algorithm to scheduling in turning processes for minimizing makespan and carbon footprint. Journal of Cleaner Production 101, 337-347.

Liu, C.G., Yang, J., Lian, J., Li, W.J., Evans, S., Yin, Y., 2014. Sustainable performance oriented operational decision-making of single machine systems with deterministic product arrival time. Journal of Cleaner Production 85 , 318-330.

Modiri-Delshad, M., Abd Rahim, N., 2014. Solving non-convex economic dispatch problem via backtracking search algorithm. Energy 77, 372-381.

Mori, M., Fujishima, M., Inamasu, Y., Oda, Y., 2011. A study on energy efficiency improvement for machine tools. Cirp Ann-Manuf Techn 60, 145-148.

Mouzon, G., Yildirim, M.B., Twomey, J., 2007. Operational methods for minimization of energy consumption of manufacturing equipment. Int. J. Prod. Res. 45, 4247-4271.

Onwubolu, G.C., Kumalo, T., 2001. Optimization of multipass turning operations with genetic algorithms. Int. J. Prod. Res. 39, 3727-3745.

Qingfu, Z., Hui, L., 2007. MOEA/D: A Multiobjective Evolutionary Algorithm Based on Decomposition. Evolutionary Computation, IEEE Transactions on 11, 712-731.

Rahimifard, S., Seow, Y., Childs, T., 2010. Minimising Embodied Product Energy to support energy efficient manufacturing. CIRP Annals - Manufacturing Technology 59, 25-28.

Rajemi, M.F., Mativenga, P.T., Aramcharoen, A., 2010. Sustainable machining: selection of optimum turning conditions based on minimum energy considerations. Journal of Cleaner Production 18, 1059-1065.

Ri-yao, C., 1984. Metal cutting principle. Machine Industry Publications China, China.

Shi, J., Liu, F., Xu, D., Chen, G., 2009. Decision model and practical method of energy-saving in NC machine tool. China Mechanical Engineering 20, 1344-1346.

Shin, Y., Joo, Y., 1992. Optimization of machining conditions with practical constraints. The International Journal of Production Research 30, 2907-2919.

Sierra, M., Coello Coello, C., 2005. Improving PSO-Based Multi-objective Optimization Using Crowding, Mutation and $\in$-Dominance, in: Coello Coello, C., Hernández Aguirre, A., Zitzler, E. (Eds.), Evolutionary Multi-Criterion Optimization. Springer Berlin Heidelberg, pp. 505-519.

Solimanpur, M., Ranjdoostfard, F., 2009. Optimisation of cutting parameters using a multi-objective genetic algorithm. Int. J. Prod. Res. 47, 6019-6036.

Tang, L.X., Wang, X.P., 2013. A Hybrid Multiobjective Evolutionary Algorithm for Multiobjective Optimization Problems. IEEE Trans. Evol. Comput. 17, 20-45.

Velchev, S., Kolev, I., Ivanov, K., Gechevski, S., 2014. Empirical models for specific energy consumption and optimization of cutting parameters for minimizing energy consumption during turning. Journal of Cleaner Production 80, 139-149.

Wang, Q., Liu, F., Wang, X., 2014. Multi-objective optimization of machining parameters considering energy consumption. Int J Adv Manuf Technol 71, 1133-1142.

Wang, S., Lu, X., Li, X.X., Li, W.D., 2015. A systematic approach of process planning and scheduling optimization for sustainable machining. Journal of Cleaner Production 87, 914-929.

Wang, X.K., 2006. Mechanical processing handbook. China machine press, Beijing.

Weiss, E.B., 1992. United Nations Conference on Environment and Development. International Legal Materials, 814-817.

Winter, M., Li, W., Kara, S., Herrmann, C., 2014. Determining optimal process parameters to increase the eco-efficiency of grinding processes. Journal of Cleaner Production 66, 644-654.

Wu, Q., Gao, L., Li, X., Zhang, C., Rong, Y., 2012. Applying an electromagnetism-like mechanism algorithm on parameter 
optimisation of a multi-pass milling process. Int. J. Prod. Res. 51, 1777-1788.

Yan, J., Li, L., 2013. Multi-objective optimization of milling parameters - the trade-offs between energy, production rate and cutting quality. Journal of Cleaner Production 52, 462-471.

Yang, S.Z., 2011. Mechanical processing handbook part II. China machine press, Beijing.

Yildirim, M.B., Mouzon, G., 2012. Single-Machine Sustainable Production Planning to Minimize Total Energy Consumption and Total Completion Time Using a Multiple Objective Genetic Algorithm. leee T Eng Manage 59, 585-597.

Yildiz, A.R., 2012. A comparative study of population-based optimization algorithms for turning operations. Inform Sciences 210, 81-88.

Yildiz, A.R., 2013. Hybrid Taguchi-differential evolution algorithm for optimization of multi-pass turning operations. Appl. Soft. Comput. 13, 1433-1439.

Yun, Y., 2014. Research on Turning Parameters Multi-objective Optimization for High-Efficiency and Low-Carbon Machining. Dalian Maritime University.

Zapotecas Martinez, S., Coello Coello, C.A., 2014. A multi-objective evolutionary algorithm based on decomposition for constrained multi-objective optimization, Evolutionary Computation (CEC), 2014 IEEE Congress on, pp. 429-436.

Zitzler, E., Thiele, L., 1999. Multiobjective evolutionary algorithms: A comparative case study and the Strength Pareto approach. IEEE Trans. Evol. Comput. 3, 257-271.

Caption list

Fig. 1 A general structure of BSA (Civicioglu, 2013)

Fig. 2 Non-dominated levels

Fig. 3 Construction of population $\mathrm{P}_{\mathrm{t}+1}$

Fig. 4 Pareto front approximations with the best IGD metric by different multi-objective algorithms on different instances (a) $d_{t}=4$, (b) $d_{t}=5$, (c) $d_{t}=6$, (d) $d_{t}=7$, (e) $d_{t}=8$, (f) $d_{t}=9$

Fig. 5 Boxplot study on IGD metric for each instance (a) $d_{t}=4$, (b) $d_{t}=5$, (c) $d_{t}=6$, (d) $d_{t}=7$, (e) $d_{t}=8$, (f) $d_{t}=9$

Fig. 6 The distribution of Pareto front for energy consumption and machining precision using MOBSA algorithm (a) $d_{t}=4$, (b) $d_{t}=5$, (c) $d_{t}=6$, (d) $d_{t}=7$, (e) $d_{t}=8$, (f) $d_{t}=9$

Fig. 7. The energy consumption and machining precision using MOBSA algorithm with 16 different combinations of $V_{\mathrm{r}}$ and $f_{\mathrm{r}}$ on point $\mathrm{B}$ for instance one (a) energy consumption, (b) machining precision

Fig. 8. The energy consumption and machining precision using MOBSA algorithm with 25 different combinations of $V_{\mathrm{s}}$ and $f_{\mathrm{s}}$ on point $\mathrm{B}$ for instance one (a) energy consumption, (b) machining precision

Table 1 Data for cutting tool

Table 2 Data for the optimization variables

Table 3 Data for the example of multi-pass turning

Table 4 Performance comparisons among MOBSA and other algorithms considered on each instance

Table 5 Specifications of three sample design points A-C in Pareto optimal fronts for different values of $d_{t}$ 